\title{
On continuity of quasi-morphisms for symplectic maps
}

\author{
Dedicated to Oleg Viro on the occasion of his 60th birthday \\ Michael Entov ${ }^{a}$, Leonid Polterovich ${ }^{b}$, Pierre Py \\ with an appendix by Michael Khanevsky
}

April 2009

\begin{abstract}
We discuss $C^{0}$-continuous homogeneous quasi-morphisms on the identity component of the group of compactly supported symplectomorphisms of a symplectic manifold. Such quasi-morphisms extend to the $C^{0}$-closure of this group inside the homeomorphism group. We show that for standard symplectic balls of any dimension, as well as for compact oriented surfaces, other than the sphere, the space of such quasi-morphisms is infinite-dimensional. In the case of surfaces, we give a user-friendly topological characterization of such quasimorphisms. We also present an application to Hofer's geometry on the group of Hamiltonian diffeomorphisms of the ball.
\end{abstract}

\section{Contents}

1 Introduction and main results $\quad \mathbf{2}$

1.1 Quasi-morphisms on groups of symplectic maps . . . . . . . 2

1.2 Detecting continuity ................. . . . . 4

1.3 Calabi homomorphism and continuity on surfaces . . . . . . 6

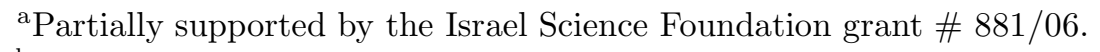

${ }^{b}$ Partially supported by the Israel Science Foundation grant \# 509/07. 
1.4 An application to Hofer's geometry . . . . . . . . . . . . 8

1.5 Acknowledgements . . . . . . . . . . . . . . . 9 9

2 Quasi-morphisms for the ball 10

3 Proof of the criterion of continuity on surfaces 12

3.1 A $C^{0}$-small fragmentation theorem on surfaces . . . . . . . . . 12

3.2 Proof of Theorem 1.7 and Corollary 1.8 . . . . . . . . . . . . 12

4 Examples of continuous quasi-morphisms $\quad 14$

5 Discussion and open questions $\quad \mathbf{1 5}$

5.1 Is $\mathcal{H}\left(\mathbb{D}^{2}\right)$ simple? (Le Roux's work) . . . . . . . . . . . . . . . 15

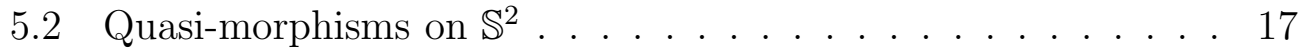

5.3 Quasi-morphisms in higher dimensions . . . . . . . . . 18

6 Proof of the fragmentation theorem 19

6.1 Preliminaries . . . . . . . . . . . . . . . . . . 19

6.2 Construction of the fragmentation . . . . . . . . . . 21

6.3 Extension lemmas . . . . . . . . . . . . . . . 23

6.4 Proof of the smooth extension lemma . . . . . . . . . . 28

7 Appendix by Michael Khanevsky:

An extension lemma for curves

\section{Introduction and main results}

\subsection{Quasi-morphisms on groups of symplectic maps}

Let $(\Sigma, \omega)$ be a compact connected symplectic manifold (possibly with a non-empty boundary $\partial \Sigma$ ). Denote by $\mathcal{D}(\Sigma, \omega)$ the identity component of the group of symplectic $C^{\infty}$-diffeomorphisms of $\Sigma$ whose supports lie in the interior of $\Sigma$. Write ${ }^{\mathrm{a}} \mathcal{H}(\Sigma, \omega)$ for the $C^{0}$-closure of $\mathcal{D}(\Sigma, \omega)$ in the group of homeomorphisms of $\Sigma$ supported in the interior of $\Sigma$. We always equip $\Sigma$ with a distance $d$ induced by a Riemannian metric on $\Sigma$, and view the

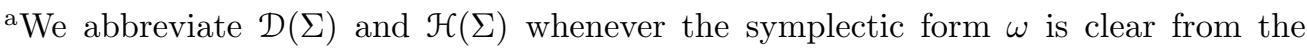
context.
} 
$C^{0}$-topology on the group of homeomorphisms of $\Sigma$ as the topology defined by the metric $\operatorname{dist}(\phi, \psi)=\max _{x \in \Sigma} d\left(x, \psi^{-1} \phi(x)\right)$.

The study of the algebraic structure of the groups $\mathcal{D}(\Sigma, \omega)$ was pioneered by Banyaga, see $[2,4]$. For instance, when $\Sigma$ is closed, he calculated the commutator subgroup of $\mathcal{D}(\Sigma, \omega)$ and showed that it is simple. However, the algebraic structure of the groups $\mathcal{H}(\Sigma, \omega)$ is much less understood. Even for the standard two-dimensional disc $\mathbb{D}^{2}$ it is still unknown whether $\mathcal{H}\left(\mathbb{D}^{2}\right)$ coincides with its commutator subgroup or not (see [10] for a comprehensive discussion). In the present paper we focus on the following algebraic feature of the groups $\mathcal{H}(\Sigma, \omega)$.

Recall that a homogeneous quasi-morphism on a group $\Gamma$ is a map $\mu$ : $\Gamma \rightarrow \mathbf{R}$ which satisfies the following two properties:

(i) There exists a constant $C(\mu) \geq 0$ such that $|\mu(x y)-\mu(x)-\mu(y)| \leq C(\mu)$ for any $x, y$ in $\Gamma$.

(ii) $\mu\left(x^{n}\right)=n \mu(x)$ for any $x \in \Gamma$ and $n \in \mathbf{Z}$.

Let us recall two well-known properties of homogeneous quasi-morphisms which will be useful in the sequel: they are invariant under conjugation, and their restrictions to abelian subgroups are homomorphisms.

The space of all homogeneous quasi-morphisms is an important algebraic invariant of the group. Quasi-morphisms naturally appear in the theory of bounded cohomology and are crucial in the study of the commutator length [6]. We refer to [6], [14], [23] or [27] for a more detailed introduction to the theory of quasi-morphisms.

Recently, several authors discovered that certain groups of diffeomorphisms preserving a volume or a symplectic form carry homogeneous quasimorphisms, see $[5,7,17,18,19,22,40,43,44]$. However, in many cases explicit constructions of non-trivial quasi-morphisms on $\mathcal{D}(\Sigma, \omega)$ require certain smoothness in an essential manner. Nevertheless, as we shall show below, some homogeneous quasi-morphisms can be extended from $\mathcal{D}(\Sigma, \omega)$ to $\mathcal{H}(\Sigma, \omega)$.

Our first result deals with the case of the Euclidean unit ball $\mathbb{D}^{2 n}$ in the standard symplectic linear space.

Theorem 1.1. The space of homogeneous quasi-morphisms on $\mathcal{H}\left(\mathbb{D}^{2 n}\right)$ is infinite-dimensional. 
The proof is given in Section 2. Next, we focus on the case when $\Sigma$ is a compact connected surface equipped with an area form. Note that in this case $\mathcal{H}(\Sigma)$ coincides with the identity component of the group of all areapreserving homeomorphisms supported in the interior of $\Sigma$, see [39] or [47].

Theorem 1.2. Let $\Sigma$ be a compact connected oriented surface other than the sphere $\mathbb{S}^{2}$, equipped with an area form. The space of homogeneous quasimorphisms on $\mathcal{H}(\Sigma)$ is infinite-dimensional.

The proof is given in Section 4. This result is new, for instance, in the case of the 2-torus. The case of the sphere is still out of reach - see Section 5.2 for a discussion. Interestingly enough, for balls of any dimension and for the twodimensional annulus, all our examples of homogeneous quasi-morphisms on $\mathcal{H}$ are based on Floer theory. When $\Sigma$ is of genus greater than one, the group $\mathcal{H}(\Sigma)$ carries a lot of homogeneous quasi-morphisms, and the statement of Theorem 1.2 readily follows from the work of Gambaudo and Ghys [22].

As an immediate application, Theorems 1.1 and 1.2 yield that if $\Sigma$ is a ball or a closed oriented surface other than sphere, then the stable commutator length is unbounded on the commutator subgroup of $\mathcal{H}(\Sigma)$. This is a standard consequence of Bavard's theory [6].

\subsection{Detecting continuity}

A key ingredient of our approach is the following proposition, due to Shtern [46]. It is a simple (nonlinear) analogue of the fact that linear forms on a topological vector space are continuous if and only if they are bounded in a neighborhood of the origin.

Proposition $1.3([46])$. Let $\Gamma$ be a topological group and $\mu: \Gamma \rightarrow \mathbf{R}$ a homogeneous quasi-morphism. Then $\mu$ is continuous if and only if it is bounded on a neighborhood of the identity.

Proof. We only prove the "if" part. Assume that $|\phi|$ is bounded by $K>0$ on an open neighborhood $\mathcal{U}$ of the identity. Let $g \in \Gamma$. For each $p \in \mathbf{N}$ define

$$
\mathcal{V}_{p}(g):=\left\{h \in \Gamma \mid h^{p} \in g^{p} \mathcal{U}\right\} .
$$

It is easy to see that $\mathcal{V}_{p}(g)$ is an open neighborhood of $g$. Pick any $h \in \mathcal{V}_{p}(g)$. Then $h^{p}=g^{p} f$ for some $f \in \mathcal{U}$. Therefore

$$
\left|\phi\left(h^{p}\right)-\phi\left(g^{p}\right)-\phi(f)\right| \leq C(\phi)
$$


hence

$$
|\phi(h)-\phi(g)| \leq \frac{C(\phi)+K}{p},
$$

which immediately yields the continuity of $\phi$ at $g$.

Let us discuss in more details the extension problem for quasi-morphisms. The next proposition shows that $C^{0}$-continuous homogeneous quasi-morphisms on $\mathcal{D}(\Sigma)$ extend to $\mathcal{H}(\Sigma)$.

Proposition 1.4. Let $\Lambda$ be a topological group and let $\Gamma \subset \Lambda$ be a dense subgroup. Any continuous homogeneous quasi-morphism on $\Gamma$ extends to a continuous homogeneous quasi-morphism on $\Lambda$.

Proof. Since $\mu$ is continuous, it is bounded by a constant $C>0$ on an open neighborhood $\mathcal{U}$ of the identity in $\Gamma$. Since $\mathcal{U}$ is open in $\Gamma$, there exists $\mathcal{U}^{\prime}$, open in $\Lambda$, so that $\mathcal{U}=\mathcal{U}^{\prime} \cap \Gamma$. We fix an open neighborhood $\mathcal{O}$ of the identity in $\Lambda$ so that $\mathcal{O}^{2} \subset \mathcal{U}^{\prime}$ and $\mathcal{O}=\mathcal{O}^{-1}$. Given $g \in \Lambda$ and $p \in \mathbf{N}$, define as before:

$$
\mathcal{V}_{p}(g):=\left\{h \in \Lambda \mid h^{p} \in g^{p} \mathcal{O}\right\} .
$$

Pick a sequence $\left\{h_{k}\right\}$ in $\Gamma$ so that each $h_{k}$ lies in $\mathcal{V}_{1}(g) \cap \ldots \cap \mathcal{V}_{k}(g)$. For $k \geq p$ we can write $h_{k}^{p}=g^{p} g_{k, p}\left(g_{k, p} \in \mathcal{O}\right)$. If $k_{1}, k_{2} \geq p$, we can write

$$
h_{k_{1}}^{p}=h_{k_{2}}^{p} g_{k_{2}, p}^{-1} g_{k_{1}, p}, \quad g_{k_{2}, p}^{-1} g_{k_{1}, p} \in \mathcal{U} .
$$

Hence, we have the inequality

$$
\left|\mu\left(h_{k_{1}}\right)-\mu\left(h_{k_{2}}\right)\right| \leq \frac{C+C(\mu)}{p} \quad\left(k_{1}, k_{2} \geq p\right),
$$

and $\left\{\mu\left(h_{p}\right)\right\}$ is a Cauchy sequence in $\mathbf{R}$. Denote its limit by $\mu^{\prime}(g)$. One can check easily that the definition is correct and that for any sequence $g_{i} \in \Gamma$ converging to $g \in \Lambda$ one has $\mu\left(g_{i}\right) \rightarrow \mu^{\prime}(g)$. This readily yields that the resulting function $\mu^{\prime}: \Lambda \rightarrow \mathbf{R}$ is a homogeneous quasi-morphism extending $\mu$. Its continuity follows from Proposition 1.3.

In view of this proposition all we need for the proof of Theorems 1.1 and 1.2 is to exhibit non-trivial homogeneous quasi-morphisms on $\mathcal{D}(\Sigma)$ which are continuous in the $C^{0}$-topology. This leads us to the problem of continuity of homogeneous quasi-morphisms which is highlighted in the title of the present paper.

Remark 1.5. Note that all the concrete quasi-morphisms that we know on groups of diffeomorphisms are continuous in the $C^{1}$-topology. 


\subsection{Calabi homomorphism and continuity on surfaces}

It is a classical fact that the Calabi homomorphism is not continuous in the $C^{0}$-topology, see [21]. We will discuss the example of the unit ball in $\mathbf{R}^{2 n}$ and then explain why the reason for the discontinuity of the Calabi homomorphism is, in a sense, universal.

First, let us recall the definition of the group of Hamiltonian diffeomorphisms of a symplectic manifold $(\Sigma, \omega)$. Given a smooth function $F$ : $\Sigma \times S^{1} \rightarrow \mathbf{R}$, supported in Interior $(\Sigma) \times S^{1}$, consider the time-dependent vector field $\operatorname{sgrad} F_{t}$ given by $i_{\mathrm{sgrad} F t}=-d F_{t}$, where $F_{t}(x)$ stands for $F(x, t)$. The flow $f_{t}$ of this vector field is called the Hamiltonian flow generated by the Hamiltonian function $F$ and its time one map $f_{1}$ is called the Hamiltonian diffeomorphism generated by $F$. Hamiltonian diffeomorphisms form a normal subgroup of $\mathcal{D}(\Sigma, \omega)$ denoted by $\operatorname{Ham}(\Sigma, \omega)$, or just by $\operatorname{Ham}(\Sigma)$. The quotient $\mathcal{D}(\Sigma) / \operatorname{Ham}(\Sigma)$ is isomorphic to the group $H_{\text {comp }}^{1}(\Sigma, \mathbf{R})$. In particular, $\mathcal{D}(\Sigma)=\operatorname{Ham}(\Sigma)$ for $\Sigma=\mathbb{D}^{2 n}$ or for $\Sigma=\mathbb{S}^{2}$. We refer to [37] for the details.

Example 1.6. Let $\Sigma=\mathbb{D}^{2 n}$ be the closed unit ball in $\mathbf{R}^{2 n}$ equipped with the symplectic form $\omega=d p \wedge d q$. Take any diffeomorphism $f \in \operatorname{Ham}\left(\mathbb{D}^{2 n}\right)$ and pick a Hamiltonian $F$ generating $f$. The value

$$
\operatorname{Cal}(f):=\int_{0}^{1} \int_{\mathbb{D}^{2 n}} F(p, q, t) d p d q d t
$$

depends only on $f$ and defines the Calabi homomorphism $C a l: \mathcal{D}\left(\mathbb{D}^{2 n}\right) \rightarrow \mathbf{R}$ [13].

Take a sequence of time-independent Hamiltonians $F_{i}$ supported in balls of radii $\frac{1}{i}$ so that $\int_{\mathbb{D}^{2 n}} F_{i} d p d q=1$. The corresponding Hamiltonian diffeomorphisms $f_{i} C^{0}$-converge to the identity and satisfy $\operatorname{Cal}\left(f_{i}\right)=1$. We conclude that the Calabi homomorphism is discontinuous in the $C^{0}$-topology.

In the remainder of this section, let us return to the case when $\Sigma$ is a compact connected surface equipped with an area form. Our next result shows, roughly speaking, that for a quasi-morphism $\mu$ on $\operatorname{Ham}(\Sigma)$ its nonvanishing on a sequence of Hamiltonian diffeomorphisms $f_{i}$ supported in a collection of shrinking balls is the only possible reason for discontinuity. The next remark is crucial for understanding this phenomenon. Observe that $\operatorname{support}\left(f^{N}\right) \subset \operatorname{support}(f)$ for any diffeomorphism $f$. Thus in the statement above non-vanishing yields unboundedness: if $\mu\left(f_{i}\right) \neq 0$ for all $i$ then the sequence $\mu\left(f_{i}^{N_{i}}\right)=N_{i} \mu\left(f_{i}\right)$ is unbounded for an appropriate choice of $N_{i}$. 
Theorem 1.7. Let $\mu: \operatorname{Ham}(\Sigma) \rightarrow \mathbf{R}$ be a homogeneous quasi-morphism. Then $\mu$ is continuous in the $C^{0}$-topology if and only if there exists a $>0$ such that the following property holds: For any disc $D \subset \Sigma$ of area less than a the restriction of $\mu$ to the group $\operatorname{Ham}(D)$ vanishes.

Here by a disc in $\Sigma$ we mean the image of a smooth embedding $\mathbb{D}^{2} \hookrightarrow \Sigma$. We view it as a surface with boundary equipped with the area form which is the restriction of the area form on $\Sigma$. The "only if" part of the theorem is elementary. It extends to certain four-dimensional symplectic manifolds (see Remark 3.2 below). The proof of the "if" part is more involved and no extension to higher dimensions is available to us so far (see the discussion in Section 5.3 below).

Corollary 1.8. Let $\mu: \mathcal{D}(\Sigma) \rightarrow \mathbf{R}$ be a homogeneous quasi-morphism. Suppose that

(i) There exists a $>0$ such that for any disc $D \subset \Sigma$ of area less than a the restriction of $\mu$ to the group $\operatorname{Ham}(D)$ vanishes.

(ii) The restriction of $\mu$ to each one-parameter subgroup of $\mathcal{D}(\Sigma)$ is linear.

Then $\mu$ is continuous in the $C^{0}$-topology.

Note that assumption (ii) is indeed necessary, provided one believes in the axiom of choice. Indeed, assuming that $\Sigma$ is not $\mathbb{D}^{2}, \mathbb{S}^{2}$ or $\mathbb{T}^{2}$, the quotient $\mathcal{D}(\Sigma) / \operatorname{Ham}(\Sigma)$ is isomorphic to the additive group of a vector space $V:=H_{\text {comp }}^{1}(\Sigma, \mathbf{R}) \neq\{0\}$. Define a quasi-morphism $\mu: \mathcal{D}(\Sigma) \rightarrow \mathbf{R}$ as the composition of the projection $\mathcal{D}(\Sigma) \rightarrow V$ with a discontinuous homomorphism $V \rightarrow \mathbf{R}$. The homomorphism $\mu$ satisfies (i), since it vanishes on $\operatorname{Ham}(\Sigma)$, and it is obviously discontinuous.

The criterion of continuity stated in Theorem 1.7 and Corollary 1.8 are proved in Section 3. They will be used in Section 4 in order to verify $C^{0}$ continuity of a certain family of quasi-morphisms on $\mathcal{D}\left(\mathbb{T}^{2}\right)$ introduced in [22] and explored in [45], which will enable us to complete the proof of Theorem 1.2. 


\subsection{An application to Hofer's geometry}

Here we concentrate on the case of the unit ball $\mathbb{D}^{2 n} \subset \mathbf{R}^{2 n}$. For a diffeomorphism $f \in \operatorname{Ham}\left(\mathbb{D}^{2 n}\right)$ define its Hofer's norm [25] as

$$
\|f\|_{H}:=\inf \int_{0}^{1}\left(\max _{z \in \mathbb{D}^{2 n}} F(z, t)-\min _{z \in \mathbb{D}^{2 n}} F(z, t)\right) d t,
$$

where the infimum is taken over all Hamiltonian functions $F$ generating $f$. Hofer's famous result states that $d_{H}(f, g):=\left\|f g^{-1}\right\|_{H}$ is a non-degenerate bi-invariant metric on $\operatorname{Ham}\left(\mathbb{D}^{2 n}\right)$. It is called Hofer's metric. It turns out that the quasi-morphisms that we construct in the proof of Theorem 1.1 are Lipschitz with respect to Hofer's metric. Hence, our proof of Theorem 1.1 yields:

Proposition 1.9. The space of homogeneous quasi-morphisms on the group $\operatorname{Ham}\left(\mathbb{D}^{2 n}\right)$ which are both continuous for the $C^{0}$-topology and Lipschitz for Hofer's metric is infinite dimensional.

The relation between Hofer's metric and the $C^{0}$-metric on $\operatorname{Ham}(\Sigma)$ is subtle. First of all, the $C^{0}$-metric is never continuous with respect to Hofer's metric. Furthermore, arguing as in Example 1.6 one can show that Hofer's metric on $\operatorname{Ham}\left(\mathbb{D}^{2 n}\right)$ is not continuous in the $C^{0}$-topology. However, for $\mathbf{R}^{2 n}$ equipped with the standard symplectic form $d p \wedge d q$ (informally speaking, this corresponds to the case of ball of infinite radius), Hofer's metric is continuous for the $C^{0}$-Whitney topology [26].

An attempt to understand the relation between Hofer's metric and the $C^{0}$-metric led Le Roux [33] to the following problem. Let $\mathscr{E}_{C} \subset \operatorname{Ham}\left(\mathbb{D}^{2 n}\right)$ be the complement of the closed ball (in Hofer's metric) of radius $C$ centered at the identity:

$$
\mathscr{E}_{C}:=\left\{f \in \operatorname{Ham}\left(\mathbb{D}^{2 n}\right), d_{H}(f, \mathbb{1})>C\right\} .
$$

Le Roux asked the following: is it true that $\mathscr{E}_{C}$ has a non-empty interior in the $C^{0}$-topology for any $C>0$ ?

The energy-capacity inequality [25] states that if $f \in \operatorname{Ham}\left(\mathbb{D}^{2 n}\right)$ displaces $\phi\left(\mathbb{D}^{2 n}(r)\right)$, where $\phi$ is any symplectic embedding of the Euclidean ball of radius $r$, then Hofer's norm of $f$ is at least $\pi r^{2}$. (We say that $f$ displaces a set $U$, if $f(U) \cap \bar{U}=\emptyset)$. By Gromov's packing inequality [24], this could happen only when $r^{2} \leq 1 / 2$. Since the displacement property is $C^{0}$-robust, we get 
that $\mathscr{E}_{C}$ indeed has a non-empty interior in the $C^{0}$-sense for $C<\pi / 2$. Using our quasi-morphisms we get an affirmative answer to Le Roux's question even for large values of $C$.

Corollary 1.10. For any $C>0$ the set $\mathscr{E}_{C}$ has a non-empty interior in the $C^{0}$-topology.

Proof. This follows just from the existence of a nontrivial homogeneous quasimorphism $\mu: \operatorname{Ham}\left(\mathbb{D}^{2 n}\right) \rightarrow \mathbf{R}$ which is both continuous in the $C^{0}$-topology and Lipschitz with respect to Hofer's metric. Indeed, pick a diffeomorphism $f$ such that

$$
\frac{|\mu(f)|}{\operatorname{Lip}(\mu)} \geq C+1
$$

where $\operatorname{Lip}(\mu)$ is the Lipschitz constant of $\mu$ with respect to Hofer's metric. There is a neighborhood $O$ of $f$ in $\operatorname{Ham}\left(\mathbb{D}^{2 n}\right)$ in the $C^{0}$-topology on which $|\mu|>C \cdot \operatorname{Lip}(\mu)$. We get that $\|g\|_{H}>C$ for $g \in O$ and hence $O \subset \mathscr{E}_{C}$. This proves the corollary.

Note that Le Roux's question makes sense on any symplectic manifold. For certain closed symplectic manifolds with infinite fundamental group one can easily get a positive answer using the energy-capacity inequality in the universal cover (as in [32]). However, for closed simply connected manifolds (and already for the case of the 2-sphere) the question is wide open.

\subsection{Acknowledgements}

This text started as an attempt to understand a remark of Dieter Kotschick. We thank him for stimulating discussions and in particular for communicating to us the idea of getting the continuity from the $C^{0}$-fragmentation, which appeared in a preliminary version of [28]. The authors would like to thank warmly Frédéric Le Roux for his comments on this work and for the thrilling discussions we had during the preparation of this article. The third author would like to thank Tel-Aviv University for its hospitality during the spring of 2008, when this work started. The second author expresses his deep gratitude to Oleg Viro for a generous help and support at the beginning of his research in topology. 


\section{Quasi-morphisms for the ball}

In this section we prove Theorem 1.1.

Denote by $\mathbb{D}^{2 n}(r)$ the Euclidean ball $\left\{|p|^{2}+|q|^{2} \leq r^{2}\right\}$, so that $\mathbb{D}^{2 n}=$ $\mathbb{D}^{2 n}(1)$. We say that a set $U$ in a symplectic manifold $(\Sigma, \omega)$ is displaceable, if there exists $\phi \in \operatorname{Ham}(\Sigma)$ which displaces it: $\phi(U) \cap \bar{U}=\emptyset$. A quasimorphism $\mu: \operatorname{Ham}(\Sigma) \rightarrow \mathbf{R}$ will be called Calabi, if for any displaceable domain $U \subset M$, such that $\left.\omega\right|_{U}$ is exact, one has $\left.\mu\right|_{\operatorname{Ham}(U)}=\left.C a l\right|_{\operatorname{Ham}(U)}$.

We will use the following result, established in [18]: there exists $a>0$ so that the group $\operatorname{Ham}\left(\mathbb{D}^{2 n}(1+a)\right)$ admits an infinite-dimensional space of quasi-morphisms which are Lipschitz in Hofer's metric, vanish on $\operatorname{Ham}(U)$ for every displaceable domain $U \subset \mathbb{D}^{2 n}(1+a)$ and do not vanish on $\operatorname{Ham}\left(\mathbb{D}^{2 n}\right)$. These quasi-morphisms are obtained by subtracting the appropriate multiple of the Calabi homomorphism from the Calabi quasi-morphisms constructed in [9]. We claim that the restriction of each such quasi-morphism, say $\eta$, to $\operatorname{Ham}\left(\mathbb{D}^{2 n}\right)$ is continuous in the $C^{0}$-topology. By Proposition 1.4, this would yield the desired result. By Proposition 1.3 it suffices to show that for some $\epsilon>0$ the quasi-morphism $\eta$ is bounded on all $f \in \operatorname{Ham}\left(\mathbb{D}^{2 n}\right)$ such that

$$
|f(x)-x|<\epsilon \forall x \in \mathbb{D}^{2 n} .
$$

For $c>0$ define the strip

$$
\Pi(c):=\left\{(p, q) \in \mathbf{R}^{2 n}:\left|q_{n}\right|<c\right\} .
$$

Choose $\epsilon>0$ so small that $\Pi(2 \epsilon) \cap \mathbb{D}^{2 n}$ is displaceable in $\mathbb{D}^{2 n}(1+a)$. Put $D_{ \pm}:=\mathbb{D}^{2 n} \cap\left\{ \pm q_{n}>0\right\}$. Observe that $D_{ \pm}$are displaceable in $\mathbb{D}^{2 n}(1+a)$ by a Hamiltonian diffeomorphism that can be represented outside a neighborhood of the boundary as a small vertical shift along the $q_{n}$-axis (in the case of $D_{+}$ we take the shift that moves it up and in the case of $D_{-}$the shift that moves it down) composed with a 180 degrees rotation in the $\left(p_{n}, q_{n}\right)$-coordinate plane. The desired boundedness result immediately follows from the next fragmentation-type lemma:

Lemma 2.1. Assume that $f \in \operatorname{Ham}\left(\mathbb{D}^{2 n}\right)$ satisfies (1). Then $f$ can be decomposed as $\theta \phi_{+} \phi_{-}$, where $\theta \in \operatorname{Ham}\left(\Pi(2 \epsilon) \cap \mathbb{D}^{2 n}\right)$ and $\phi_{ \pm} \in \operatorname{Ham}\left(D_{ \pm}\right)$.

Indeed, $\eta$ vanishes on $\operatorname{Ham}(U)$ for every displaceable domain $U \subset \mathbb{D}^{2 n}(1+a)$. Since $\Pi(2 \epsilon) \cap \mathbb{D}^{2 n}$ and $D_{ \pm}$are displaceable, $\eta(\theta)=\eta\left(\phi_{ \pm}\right)=0$. Thus $|\eta(f)| \leq$ 
$2 C(\eta)$ for every $f \in \operatorname{Ham}\left(\mathbb{D}^{2 n}\right)$ lying in the $\epsilon$-neighborhood of the identity with respect to the $C^{0}$-distance, and the theorem follows. It remains to prove the lemma.

Proof of Lemma 2.1: Denote by $S$ the hyperplane $\left\{q_{n}=0\right\}$. For $c>0$ write $R_{c}$ for the dilation $z \rightarrow c z$ of $\mathbf{R}^{2 n}$. We assume that all compactly supported diffeomorphisms of $\mathbb{D}^{2 n}$ are extended to the whole $\mathbf{R}^{2 n}$ by the identity.

Take $f \in \operatorname{Ham}\left(\mathbb{D}^{2 n}\right)$ which satisfies (1). Let $\left\{f_{t}\right\}_{0 \leq t \leq 1}$, be a Hamiltonian isotopy supported in $\mathbb{D}^{2 n}$ so that $f_{t}=\mathbb{1}$ for $t \in[0, \delta)$ and $f_{t}=f$ for $t \in$ $(1-\delta, 1]$ for some $\delta>0$. Take a smooth function $c:[0,1] \rightarrow[1,+\infty)$ which equals 1 near 0 and 1 and satisfies $c(t)>(2 \epsilon)^{-1}$ on $[\delta, 1-\delta]$. Consider the Hamiltonian isotopy $h_{t}=R_{1 / c(t)} f_{t} R_{c(t)}$ of $\mathbf{R}^{2 n}$. Note that $h_{0}=1$ and $h_{1}=f$. Since $c(t) \geq 1$, we have $h_{t} z=z$ for $z \notin \mathbb{D}^{2 n}$, and $h_{t}$ is supported in $\mathbb{D}^{2 n}$.

We claim that $h_{t}(S) \subset \Pi(2 \epsilon)$. Observe that $R_{c(t)} S=S$. Take any $z \in S$. If $R_{c(t)} z \notin \mathbb{D}^{2 n}$, we have that $h_{t} z=z$. Assume now that $R_{c(t)} z \in \mathbb{D}^{2 n}$. Consider the following cases:

- If $t \in(1-\delta, 1]$, then $f_{t} R_{c(t)}(S)=f(S)$. Thus $f_{t} R_{c(t)} z \in f\left(S \cap \mathbb{D}^{2 n}\right) \subset$ $\Pi(2 \epsilon)$, where the latter inclusion follows from (1). Therefore $h_{t} z \in$ $\Pi(2 \epsilon)$ since $c(t) \geq 1$.

- If $t \in[\delta, 1-\delta]$, then $h_{t} z \in \mathbb{D}^{2 n}(2 \epsilon) \subset \Pi(2 \epsilon)$ by our choice of the function $c(t)$.

- If $t \in[0, \delta)$, then $h_{t} S=S \subset \Pi(2 \epsilon)$.

This completes the proof of the claim.

By continuity of $h_{t}$, there exists $\kappa>0$ so that $h_{t}(\Pi(\kappa)) \subset \Pi(2 \epsilon)$ for all $t$. Cutting off the Hamiltonian of $h_{t}$ near $h_{t}(\Pi(\kappa))$ we get a Hamiltonian flow $\theta_{t}$ supported in $\Pi(2 \epsilon)$ which coincides with $h_{t}$ on $\Pi(\kappa)$. Thus, $\theta_{t}^{-1} h_{t}$ is the identity on $\Pi(\kappa)$ for all $t$. It follows that $\theta_{t}^{-1} h_{t}$ decomposes into the product of two commuting Hamiltonian flows $\phi_{-}^{t}$ and $\phi_{+}^{t}$ supported in $D_{-}$and $D_{+}$ respectively. Therefore $f=\theta_{1} \phi_{-}^{1} \phi_{+}^{1}$ is the desired decomposition. 


\section{Proof of the criterion of continuity on sur- faces}

\subsection{A $C^{0}$-small fragmentation theorem on surfaces}

Before stating our next result we recall the notion of fragmentation of a diffeomorphism. This is a classical technique in the study of groups of diffeomorphisms, see e.g. $[2,4,10]$. Given a Hamiltonian diffeomorphism $f$ of a connected symplectic manifold $\Sigma$, and an open cover $\left\{U_{\alpha}\right\}$ of $\Sigma$, one can always write $f$ as a product of Hamiltonian diffeomorphisms each of which is supported in one of the open sets $U_{\alpha}$. It is known that the number of factors in such a decomposition is uniform in a $C^{1}$-neighborhood of the identity, see $[2,4,10]$. To prove our continuity theorem we actually need to prove a similar result on surfaces when one consider diffeomorphisms endowed with the $C^{0}$-topology. Such a result appears in [34] in the case when the surface is the unit disc. Observe also that the corresponding fragmentation result is known for volume-preserving homeomorphisms [20].

Theorem 3.1. Let $\Sigma$ be a compact connected surface (possibly with boundary), equipped with an area-form. Then for every $a>0$ there exists a neighborhood $\mathcal{U}$ of the identity in the group $\operatorname{Ham}(\Sigma)$ endowed with the $C^{0}$-topology and an integer $N>0$ such that any diffeomorphism $g \in \mathcal{U}$ can be written as a product of at most $N$ Hamiltonian diffeomorphisms supported in discs of area less than $a$.

This result might be well-known to experts and probably can be deduced from the corresponding result for homeomorphisms. However, since the proof is more difficult for homeomorphisms, and in order to keep this paper selfcontained, we are going to give a direct proof of Theorem 3.1 in Section 6 . Note that this last section is the most technical part of the text. Given the fragmentation result above, one obtains easily a proof of Theorem 1.7, as we will show now.

\subsection{Proof of Theorem 1.7 and Corollary 1.8}

1)We begin by proving that the condition appearing in the statement of the theorem is necessary for the quasi-morphism $\mu$ to be continuous. Assume $\mu$ is continuous for the $C^{0}$-topology. Then it is bounded on some 
$C^{0}$-neighborhood $\mathcal{U}$ of the identity in $\operatorname{Ham}(\Sigma)$. Choose now a disc $D_{0}$ in $\Sigma$. If $D_{0}$ has a sufficiently small diameter, then $\operatorname{Ham}\left(D_{0}\right) \subset \mathcal{U}$. But since $\operatorname{Ham}\left(D_{0}\right)$ is a subgroup and $\mu$ is homogeneous, $\mu$ must vanish on $\operatorname{Ham}\left(D_{0}\right)$.

Now, let $a=\operatorname{area}\left(D_{0}\right)$. If $D$ is any disc of area less than $a$, the group $\operatorname{Ham}(D)$ is conjugated in $\operatorname{Ham}(\Sigma)$ to a subgroup of $\operatorname{Ham}\left(D_{0}\right)$, because for any two discs of the same area in $\Sigma$ there exists a Hamiltonian diffeomorphism mapping one of the discs onto another - see e.g. [1], Proposition A.1, for a proof (which, in fact, works for all $\Sigma$, though the claim there is stated only for closed surfaces). Hence, $\mu$ vanishes on $\operatorname{Ham}(D)$ as required.

Remark 3.2. This proof extends verbatim to higher-dimensional symplectic manifolds $(\Sigma, \omega)$ which admit a positive constant $a_{0}$ with the following property: for every $a<a_{0}$ all symplectically embedded balls of volume $a$ in the interior of $\Sigma$ are Hamiltonian isotopic. Here a symplectically embedded ball of volume $a$ is the image of the standard Euclidean ball of volume $a$ in $\left(\mathbf{R}^{2 n}, d p \wedge d q\right)$ under a symplectic embedding. This property holds for instance for blow-ups of rational and ruled symplectic four-manifolds, see $[35,29,8,36]$.

2) We now prove the reverse implication. Assume that a homogeneous quasi-morphism $\mu$ vanishes on all Hamiltonian diffeomorphisms supported in discs of area $<a$. Take the $C^{0}$-neighborhood $\mathcal{U}$ of the identity and the integer $N$ from Theorem 3.1. Then $\mu$ is bounded by $(N-1) C(\mu)$ on $\mathcal{U}$, and hence, continuous by Proposition 1.3.

We now prove Corollary 1.8. Choose compactly supported symplectic vector fields $v_{1}, \ldots, v_{k}$ on $\Sigma$ so that the cohomology classes of the 1-forms $i_{v_{j}} \omega$ generate $H_{\text {comp }}^{1}(\Sigma, \mathbf{R})$. Denote by $h_{i}^{t}$ the flow of $v_{i}$. Let $\mathcal{V}$ be the image of the following map:

$$
\begin{aligned}
(-\epsilon, \epsilon)^{k} & \rightarrow \mathcal{D} \\
\left(t_{1}, \ldots, t_{k}\right) & \mapsto \prod_{i=1}^{k} h_{i}^{t_{i}} .
\end{aligned}
$$

Using assumption (i) and applying Theorem 1.7 we get that the quasimorphism $\mu$ is bounded on a $C^{0}$-neighborhood, say $\mathcal{U}$, of the identity in $\operatorname{Ham}(\Sigma)$. Thus by (ii) and the definition of a quasi-morphism, $\mu$ is bounded on $\mathcal{U} \cdot \mathcal{V}$. But the latter set is a $C^{0}$-neighborhood of the identity in $\mathcal{D}$. Thus $\mu$ is continuous on $\mathcal{D}$ by Proposition 1.3. 


\section{Examples of continuous quasi-morphisms}

In this section we prove case by case Theorem 1.2. The case of the disc has been already explained in Section 2. This construction generalizes verbatim to all closed surfaces of genus 0 with non-empty boundary, which proves Theorem 1.2 in this case.

When $\Sigma$ is a closed surface of genus greater than one, Gambaudo and Ghys constructed in [22] an infinite-dimensional space of homogeneous quasimorphisms on the group $\mathcal{D}(\Sigma)$, satisfying the hypothesis of Theorem 1.7. These quasi-morphisms are defined using 1-forms on the surface and can be thought of as some "quasi-fluxes". We refer to Section 6.1 of [22] or to Section 2.5 of [23] for a detailed description. The fact that these quasimorphisms extend continuously to the identity component of the group of area-preserving homeomorphisms of $\Sigma$ can be checked easily without appealing to Theorem 1.7. This was already observed in [23].

In order to settle the case of surfaces of genus one, we shall apply the criterion given by Theorem 1.7. The quasi-morphisms that we will use were constructed by Gambaudo and Ghys in [22], see also [45]. We recall briefly this construction now.

The fundamental group $\pi_{1}\left(\mathbb{T}^{2} \backslash\{0\}\right)$ of the once-punctured torus is a free group on two generators, $a$ and $b$, represented by a parallel and a meridian in $\mathbb{T}^{2} \backslash\{0\}$. Let $\mu: \pi_{1}\left(\mathbb{T}^{2} \backslash\{0\}\right) \rightarrow \mathbf{R}$ be a homogeneous quasi-morphism. It is known that there are plenty of such quasi-morphisms (see [11] for instance). We will associate to $\mu$ a homogeneous quasi-morphism $\widetilde{\mu}$ on the group $\mathcal{D}\left(\mathbb{T}^{2}\right)$.

We fix a base point $x_{*} \in \mathbb{T}^{2} \backslash\{0\}$. For all $v \in \mathbb{T}^{2} \backslash\{0\}$ we choose a path $\alpha_{v}(t), t \in[0,1]$, in $\mathbb{T}^{2} \backslash\{0\}$ from $x_{*}$ to $v$. We assume that the lengths of the paths $\alpha_{v}$ are uniformly bounded with respect to a Riemannian metric defined on the compact surface obtained by blowing-up the origin on $\mathbb{T}^{2}$. Consider an element $f \in \mathcal{D}\left(\mathbb{T}^{2}\right)$ and fix an isotopy $\left(f_{t}\right)$ from the identity to $f$. If $x$ and $y$ are distinct points in the torus, we can consider the curve

$$
f_{t}(x)-f_{t}(y)
$$

in $\mathbb{T}^{2} \backslash\{0\}$. Its homotopy class depends only on $f$. We close it to form a loop:

$$
\alpha(f, x, y):=\alpha_{x-y} *\left(f_{t}(x)-f_{t}(y)\right) * \overline{\alpha_{f(x)-f(y)}},
$$

where $\overline{\alpha_{f(x)-f(y)}}(t):=\alpha_{f(x)-f(y)}(1-t)$. We have the cocycle relation:

$$
\alpha(f g, x, y)=\alpha(g, x, y) * \alpha(f, g(x), g(y)) .
$$


Define a function $u_{f}$ on $\mathbb{T}^{2} \times \mathbb{T}^{2} \backslash \Delta$ (where $\Delta$ is the diagonal) by $u_{f}(x, y)=$ $\mu(\alpha(f, x, y))$. From the previous relation and the fact that $\mu$ is a quasimorphism we deduce the relation:

$$
\left|u_{f g}(x, y)-u_{g}(x, y)-u_{f}(g(x), g(y))\right| \leq C(\mu), \forall f, g \in \mathcal{D}\left(\mathbb{T}^{2}\right) .
$$

Moreover, it is not difficult to see that the function $u_{f}$ is measurable and bounded on $\mathbb{T}^{2} \times \mathbb{T}^{2} \backslash \Delta$. Hence, the map

$$
f \mapsto \int_{\mathbb{T}^{2} \times \mathbb{T}^{2}} u_{f}(x, y) d x d y
$$

is a quasi-morphism. We denote by $\widetilde{\mu}$ the associated homogeneous quasimorphism:

$$
\widetilde{\mu}(f)=\lim _{p \rightarrow \infty} \frac{1}{p} \int_{\mathbb{T}^{2} \times \mathbb{T}^{2}} u_{f p}(x, y) d x d y .
$$

One easily check that $\widetilde{\mu}$ is linear on any 1-parameter subgroup. The following proposition was established in [45]:

Proposition 4.1. Let $f \in \operatorname{Ham}\left(\mathbb{T}^{2}\right)$ be a diffeomorphism supported in a disc D. Then for any homogeneous quasi-morphism $\mu: \pi_{1}\left(\mathbb{T}^{2} \backslash\{0\}\right) \rightarrow \mathbf{R}$ one has

$$
\widetilde{\mu}(f)=2 \mu([a, b]) \cdot \operatorname{Cal}(f),
$$

where Cal $: \operatorname{Ham}(D) \rightarrow \mathbf{R}$ is the Calabi homomorphism.

By Corollary 1.8, we get that the quasi-morphisms $\widetilde{\mu}$, where $\mu$ runs over the set of homogeneous quasi-morphisms on $\pi_{1}\left(\mathbb{T}^{2} \backslash\{0\}\right)$ which take the value 0 on the element $[a, b]$, are all continuous in the $C^{0}$-topology. According to [22], this family spans an infinite-dimensional vector space. To complete the proof of Theorem 1.2 for surfaces of genus 1, we only have to check that the diffeomorphisms which were constructed in [22] in order to establish the existence of an arbitrary number of linearly independent quasi-morphisms $\widetilde{\mu}$ can be chosen to be supported in any given subsurface of genus one. But this follows easily from the construction in Section 6.2 of [22].

\section{Discussion and open questions}

\subsection{Is $\mathcal{H}\left(\mathbb{D}^{2}\right)$ simple? (Le Roux's work)}

Although the algebraic structure of groups of volume-preserving homeomorphisms in dimension greater than 2 is well-understood [20], the case of 
area-preserving homeomorphisms of surfaces is still mysterious. In particular, it is unknown whether the group $\mathcal{H}\left(\mathbb{D}^{2}\right)$ is simple. Some normal subgroups of $\mathcal{H}\left(\mathbb{D}^{2}\right)$ were constructed by Ghys, Oh, and more recently by Le Roux, see [10] for a survey. However, it is unknown whether any of these normal subgroups is a proper subgroup of $\mathcal{H}\left(\mathbb{D}^{2}\right)$. In [34], Le Roux established that the simplicity of the group $\mathcal{H}\left(\mathbb{D}^{2}\right)$ is equivalent to a certain fragmentation property. Namely, he established the following result (in the following we assume that the total area of the disc is 1$)$ :

The group $\mathcal{H}\left(\mathbb{D}^{2}\right)$ is simple if and only if there exist numbers $\rho^{\prime}<\rho$ in $(0,1]$ and an integer $N$ so that the following holds: any homeomorphism $g \in \mathcal{H}\left(\mathbb{D}^{2}\right)$, whose support is contained in a disc of area at most $\rho$, can be written as a product of at most $N$ homeomorphisms whose supports are contained in discs of area at most $\rho^{\prime}$.

(By a result of Fathi [20], cf. [34], g can always be represented as such a product with some, a priori unknown, number of factors).

Remark 5.1. One can show that the property above depends only on $\rho$ and not of the choice of $\rho^{\prime}$ smaller than $\rho$ [34].

In the sequel we will denote by $G_{\varepsilon}$ the set of homeomorphisms in $\mathcal{H}\left(\mathbb{D}^{2}\right)$ whose support is contained in an open disc of area at most $\varepsilon$. For an element $g \in \mathcal{H}\left(\mathbb{D}^{2}\right)$ we define (following $\left.[12,34]\right)|g|_{\varepsilon}$ as the minimal integer $n$ such that $g$ can be written as a product of $n$ homeomorphisms of $G_{\varepsilon}$. Any homogeneous quasi-morphism $\phi$ on $\mathcal{H}\left(\mathbb{D}^{2}\right)$ which vanishes on $G_{\varepsilon}$ gives the following lower bound on $|\cdot|_{\varepsilon}$ :

$$
|g|_{\varepsilon} \geq \frac{|\phi(g)|}{C(\phi)} \quad\left(g \in \mathcal{H}\left(\mathbb{D}^{2}\right)\right)
$$

In particular, if $\phi$ vanishes on $G_{\varepsilon}$ but not on $G_{\varepsilon^{\prime}}$ for some $\varepsilon^{\prime}>\varepsilon$, then the norm $|\cdot|_{\varepsilon}$ is unbounded on $G_{\varepsilon^{\prime}}$.

If $\phi: \mathcal{H}\left(\mathbb{D}^{2}\right) \rightarrow \mathbf{R}$ is a homogeneous quasi-morphism which is continuous in the $C^{0}$-topology, we can define $a(\phi)$ to be the supremum of the positive numbers $a$ satisfying the following property: $\phi$ vanishes on $\operatorname{Ham}(D)$ for any disc $D$ of area less or equal than $a$ (for a homogeneous quasi-morphism which is not continuous in the $C^{0}$-topology, one can define $\left.a(\phi)=0\right)$. One can think of $a(\phi)$ as the scale at which one can detect the nontriviality of $\phi$. According to the discussion above, the existence of a nontrivial quasi-morphism with $a(\phi)>0$ implies that the norm $|\cdot|_{a(\phi)}$ is unbounded on the set $G_{\rho}$ (for any $\rho>a(\phi))$. 
According to Le Roux's result, the existence of a sequence of continuous (for the $C^{0}$-topology) homogeneous quasi-morphisms $\phi_{n}$ on $\mathcal{H}\left(\mathbb{D}^{2}\right)$ with $a\left(\phi_{n}\right) \rightarrow 0$ would imply that the group $\mathcal{H}\left(\mathbb{D}^{2}\right)$ is not simple. However, for all the examples of quasi-morphisms on $\mathcal{H}\left(\mathbb{D}^{2}\right)$ that we know (coming from the continuous quasi-morphisms described in Section 2), one has $a(\phi) \geq \frac{1}{2}$.

\subsection{Quasi-morphisms on $\mathbb{S}^{2}$}

Consider the sphere $\mathbb{S}^{2}$ equipped with an area form of total area 1.

Question 5.2. (i) Does there exist a non-vanishing $C^{0}$-continuous homogeneous quasi-morphism on $\operatorname{Ham}\left(\mathbb{S}^{2}\right)$ ?

(ii) If yes, can it be made Lipschitz with respect to Hofer's metric?

If the answer to the first question was negative, this would imply that the Calabi quasi-morphism constructed in [18] is unique. Indeed, the difference of two Calabi quasi-morphisms is continuous in the $C^{0}$-topology according to Theorem 1.7. Note that for surfaces of positive genus, the examples of $C^{0}$-continuous quasi-morphisms that we gave are related to the existence of many Calabi quasi-morphisms [44, 45].

In turn, the affirmative answer to Question 5.2(ii) would yield the solution of the following problem posed by Misha Kapovich and the second named author in 2006. It is known [42] that $\operatorname{Ham}\left(\mathbb{S}^{2}\right)$ carries a one-parameter subgroup, say $L:=\left\{f_{t}\right\}_{t \in \mathbf{R}}$, which is a quasi-geodesic in the following sense: $\left\|f_{t}\right\|_{H} \geq c|t|$ for some $c>0$ and all $t$. Given such a subgroup, put

$$
A(L):=\sup _{\phi \in \operatorname{Ham}\left(\mathbb{S}^{2}\right)} d_{H}(\phi, L)
$$

Question 5.3. Is $A(L)$ finite or infinite?

The finiteness of $A(L)$ does not depend on the specific quasi-geodesic oneparameter subgroup $L$. Intuitively, the finiteness of $A(L)$ would yield that the whole group $\operatorname{Ham}\left(\mathbb{S}^{2}\right)$ lies in a tube of a finite radius around $L$.

We claim that if $\operatorname{Ham}\left(\mathbb{S}^{2}\right)$ admits a non-vanishing $C^{0}$-continuous homogeneous quasi-morphism, which is Lipschitz in Hofer's metric, then $A(L)=\infty$. 
Indeed, such a quasi-morphism would be independent from the Calabi quasimorphism constructed in [18]. But the existence of two independent homogeneous quasi-morphisms on $\operatorname{Ham}\left(\mathbb{S}^{2}\right)$ which are Lipschitz with respect to Hofer's metric implies that $A(L)=\infty$ : otherwise the finiteness of $A(L)$ would imply that Lipschitz homogeneous quasi-morphisms are determined by their restriction to $L$.

\subsection{Quasi-morphisms in higher dimensions}

Consider the following general question: given a homogeneous quasimorphism on $\operatorname{Ham}\left(\Sigma^{2 n}, \omega\right)$, is it continuous in $C^{0}$-topology?

The answer is positive, for instance, for quasi-morphisms coming from the fundamental group $\pi_{1}(M)[22,43]$. It would be interesting to explore, for instance, the $C^{0}$-continuity of a quasi-morphism $\mu$ given by the difference of a Calabi quasi-morphism and the Calabi homomorphism [9, 18] (or, more generally, by the difference of two distinct Calabi quasi-morphisms). In order to prove the $C^{0}$-continuity of $\mu$, one should establish a $C^{0}$-small fragmentation lemma with a controlled number of factors in the spirit of Lemma 2.1 for $\mathbb{D}^{2 n}$ or Theorem 3.1 for surfaces. It is likely that the argument which we used for $\mathbb{D}^{2 n}$ could go through without great complications for certain Liouville symplectic manifolds, that is compact exact symplectic manifolds which admit a conformally symplectic vector field transversal to the boundary, such as the open unit cotangent bundle of the sphere.

Our result for $\mathbb{D}^{2 n}$ should also allow the construction of continuous quasimorphisms for groups of Hamiltonian diffeomorphisms of certain symplectic manifolds symplectomorphic to "sufficiently large" open subsets of $\mathbb{D}^{2 n}$ (for instance, the open unit cotangent bundle of a torus).

The $C^{0}$-small fragmentation problem on general higher-dimensional manifolds looks very difficult. Consider, for instance, the following toy case: find a fragmentation with a controlled number of factors for a $C^{0}$-small Hamiltonian diffeomorphism supported in a sufficiently small ball $D \subset \Sigma$. A crucial difference from the situation described in Section 2 is that we have no information about the Hamiltonian isotopy $\left\{f_{t}\right\}$ joining $f$ with the identity: it can "travel" far away from $D$. In particular, when $\operatorname{dim} \Sigma \geq 6$, we do not know whether $f$ lies in $\operatorname{Ham}(D)$ or not. When $\operatorname{dim} \Sigma=4$, the fact that $f \in \operatorname{Ham}(D)$ (and hence the fragmentation in our toy example) follows from a deep theorem by Gromov based on pseudo-holomorphic curves techniques [24]. It would be interesting to apply powerful methods of four-dimensional 
symplectic topology to the $C^{0}$-small fragmentation problem.

\section{Proof of the fragmentation theorem}

In this section we prove Theorem 3.1. First, we need to remind a few classical results.

\subsection{Preliminaries}

In the course of the proof we will repeatedly use the following result:

Proposition 6.1. Let $\Sigma$ be a compact connected oriented surface, possibly with a non-empty boundary $\partial \Sigma$, and let $\omega_{1}, \omega_{2}$ be two area-forms on $\Sigma$. Assume that $\int_{\Sigma} \omega_{1}=\int_{\Sigma} \omega_{2}$. If $\partial \Sigma \neq \emptyset$, we also assume that the forms $\omega_{1}$ and $\omega_{2}$ coincide on $\partial \Sigma$.

Then there exists a diffeomorphism $f: \Sigma \rightarrow \Sigma$, isotopic to the identity, such that $f^{*} \omega_{2}=\omega_{1}$. Moreover, $f$ can be chosen to satisfy the following properties:

(i) If $\partial \Sigma \neq \emptyset$, then $f$ is the identity on $\partial \Sigma$, and if $\omega_{1}$ and $\omega_{2}$ coincide near $\partial \Sigma$, then $f$ is the identity near $\partial \Sigma$.

(ii) If $\Sigma$ is partitioned into polygons (with piecewise smooth boundaries), so that $\omega_{2}-\omega_{1}$ is zero on the 1-skeleton $\Gamma$ of the partition and the integrals of $\omega_{1}$ and $\omega_{2}$ over each polygon are equal, then $f$ can be chosen to be the identity on $\Gamma$.

(iii) The diffeomorphism $f$ can be chosen arbitrarily $C^{0}$-close to $\mathbb{1}$, provided $\omega_{1}$ and $\omega_{2}$ are sufficiently $C^{0}$-close to each other (i.e. $\omega_{2}=\chi \omega_{1}$ for a function $\chi$ sufficiently $C^{0}$-close to 1 ).

The existence of $f$ in the case of a closed surface follows from a wellknown theorem of Moser [38]. The method of the proof ("Moser's method") can be outlined as follows. Set $\omega_{t}:=\omega_{1}+t\left(\omega_{2}-\omega_{1}\right)$ and note that the form $\omega_{2}-\omega_{1}$ is exact. Choose a 1 -form $\sigma$ so that $d \sigma=\omega_{2}-\omega_{1}$ and define $f$ as the time- 1 flow of the vector field $\omega_{t}$-dual to $\sigma$. In order to show (i) and (ii) one has to choose a primitive $\sigma$ for $\omega_{2}-\omega_{1}$ that vanishes near $\partial \Sigma$ or, respectively, on $\Gamma$ - the construction of such a $\sigma$ can be easily extracted from [3]. Property (iii) is essentially contained in [38]: it follows easily from the above construction of $f$, provided we can construct a $C^{0}$-small primitive $\sigma$ 
for a $C^{0}$-small exact 2-form $\omega_{2}-\omega_{1}$, but, by Lemma 1 from [38], it suffices to do it on a rectangle and in this case $\sigma$ can be constructed explicitly.

In fact, a stronger result than (iii) is true. It is known, see [39, 47], that $f$ can be chosen $C^{0}$-close to the identity as soon as the two area forms (considered as measures) are close in the weak-* topology. Note that if one of the two forms is the image of the other by a diffeomorphism $C^{0}$-close to the identity, the two forms are close in the weak-* topology. However, to keep this text self-contained, we are not going to use this fact, but will reprove directly the particular cases we need.

We equip the surface $\Sigma$ with a fixed Riemannian metric and denote by $d$ the corresponding distance. For any map $f: X \rightarrow \Sigma$ (where $X$ is a closed subset of $\Sigma$ ) we denote by $\|f\|:=\max _{x} d(x, f(x))$ its $C^{0}$-norm. Accordingly, the $C^{0}$-norm of a smooth function $u$ defined on a closed subset of $\Sigma$ will be denoted by $\|u\|$.

The following lemmas are the main tools for the proof.

Lemma 6.2 (Area-preserving extension lemma for discs). Let $D_{1} \subset D_{2} \subset$ $D \subset \mathbf{R}^{2}$ be closed discs such that $D_{1} \subset$ Interior $\left(D_{2}\right) \subset D_{2} \subset$ Interior $(D)$. Let $\phi: D_{2} \rightarrow D$ be a smooth area-preserving embedding (we assume $D$ is equipped with some area form). Then there exists $\psi \in \operatorname{Ham}(D)$ such that

$$
\left.\psi\right|_{D_{1}}=\phi \quad \text { and } \quad\|\psi\| \rightarrow 0 \text { as }\|\phi\| \rightarrow 0 .
$$

Lemma 6.3 (Area-preserving extension lemma for rectangles). Let $\Pi=$ $[0, R] \times[-c, c]$ be a rectangle and let $\Pi_{1} \subset \Pi_{2} \subset \Pi$ be two smaller rectangles of the form $\Pi_{i}=[0, R] \times\left[-c_{i}, c_{i}\right](i=1,2), 0<c_{1}<c_{2}<c$. Let $\phi: \Pi_{2} \rightarrow \Pi$ be an area-preserving embedding (we assume $\Pi$ is equipped with some area form) such that

- $\phi$ is the identity near $0 \times\left[-c_{2}, c_{2}\right]$ and $R \times\left[-c_{2}, c_{2}\right]$.

- The area in $\Pi$ bounded by the curve $[0, R] \times y$ and its image under $\phi$ is zero for some (and hence for all) $y \in\left[-c_{2}, c_{2}\right]$.

Then there exists $\psi \in \operatorname{Ham}(\Pi)$ such that

$$
\left.\psi\right|_{\Pi_{1}}=\phi \quad \text { and } \quad\|\psi\| \rightarrow 0 \text { as }\|\phi\| \rightarrow 0 .
$$

The lemmas will be proved in Section 6.3. 


\subsection{Construction of the fragmentation}

We are now ready to prove the fragmentation theorem. In the case when $\Sigma$ is the closed unit disc $\mathbb{D}^{2}$ in $\mathbf{R}^{2}$ the theorem has been proved by Le Roux [34] (Proposition 4.2). In general, our proof relies on the case of the disc.

For any $b>0$ we fix a neighborhood $\mathscr{U}_{0}(b)$ of the identity in $\operatorname{Ham}\left(\mathbb{D}^{2}\right)$ and an integer $N_{0}(b)$ such that every element of $\mathscr{U}_{0}(b)$ is a product of at most $N_{0}(b)$ diffeomorphisms supported in discs of area at most $b$. We will prove the following assertion.

For any $\epsilon>0$ there exists a neighborhood $\mathscr{V}(\epsilon)$ of the identity in $\operatorname{Ham}(\Sigma)$, an integer $N_{1}(\epsilon)$ and $N_{1}(\epsilon)$ discs $\left(D_{j}\right)_{1 \leq j \leq N_{1}(\epsilon)}$ in $\Sigma$ such that any diffeomorphism $f \in \mathscr{V}(\epsilon)$ can be written as a product $f=g_{1} \cdot \ldots \cdot g_{N_{1}(\epsilon)}$, where each $g_{i}$ belongs to $\operatorname{Ham}\left(D_{j}\right)$ for one of the discs $D_{j}$ and is $\epsilon$-close to the identity. (*)

Note that there is no restriction in $(*)$ on the areas of the discs $D_{j}$. Let us explain how to conclude the proof of Theorem 3.1 from this assertion. Fix $a>0$. We can choose, for each $i$ between 1 and $N_{1}(\epsilon)$, a conformally symplectic diffeomorphism $\psi_{i}: \mathbb{D}^{2} \rightarrow D_{i}$, so that the pull-back of the area form on $\Sigma$ by $\psi_{i}$ equals the standard area form on the disc $\mathbb{D}^{2}$ times some constant $\lambda_{i}>0$. If $\epsilon$ is sufficiently small, $\psi_{i}^{-1} g_{i} \psi_{i}$ is in $\mathscr{U}_{0}\left(\frac{a}{\lambda_{i}}\right)$ for each $i$ and we can apply the result for the disc to it. This concludes the proof.

Remark 6.4. It is important that the discs $D_{i}$ as well as the maps $\psi_{i}$ are chosen in advance, since we need the neighborhoods $\psi_{i} \mathscr{U}_{0}\left(\frac{a}{\lambda_{i}}\right) \psi_{i}^{-1}$ to be known in advance. They determine the neighborhood $\mathscr{V}(\epsilon)$.

We now prove $(*)$. The arguments we use are inspired from the work of Fathi [20]. Fix $\epsilon>0$. We distinguish between two cases: 1) $\Sigma$ has a boundary, and 2) $\Sigma$ is closed.

First case. Any compact connected surface with non-empty boundary can be obtained by gluing finitely many 1-handles to a disc. We prove the statement $(*)$ by induction on the number of 1-handles. We already know that $(*)$ is true for a disc (just take $N_{1}(\epsilon)=1$ and let $D_{1}$ be the whole disc). Assume now that $(*)$ holds for any compact surface with boundary obtained by gluing $l 1$-handles to the disc. Let $\Sigma$ be a compact surface obtained by gluing a 1-handle to a compact surface $\Sigma_{0}$, where $\Sigma_{0}$ is obtained from the disc by gluing $l 1$-handles. 
Choose a diffeomorphism (singular at the corners) $\varphi:[-1,1]^{2} \rightarrow \overline{\Sigma-\Sigma_{0}}$, sending $[-1,1] \times\{-1,1\}$ into the boundary of $\Sigma_{0}$. Let $\Pi_{r}=\varphi([-1,1] \times$ $[-r, r])$. Let $\mathscr{V}_{1}(\epsilon)$ be the neighborhood of the identity in $\operatorname{Ham}\left(\Sigma_{1}\right)$, given by (*) applied to the surface $\Sigma_{1}:=\Sigma_{0} \cup \varphi\left([0,1] \times\left\{s,|s| \geq \frac{1}{4}\right\}\right)$, and let $N_{1}(\epsilon)$ be the corresponding integer.

Let $f \in \operatorname{Ham}(\Sigma),\|f\|<\epsilon$. We apply Lemma 6.3 to the chain of rectangles $\Pi_{\frac{1}{2}} \subset \Pi_{\frac{3}{4}} \subset \Pi_{\frac{7}{8}}$ and to the restriction of $f$ to $\Pi_{\frac{3}{4}}$ (the hypothesis on the curve $[-1,1] \times\{y\}$ is met because $f$ is Hamiltonian). We obtain a diffeomorphism $\psi$ supported in $\Pi_{\frac{7}{8}}$ and $C^{0}$-close to the identity, which coincides with $f$ on $\Pi_{\frac{1}{2}}$. Hence, we can write

$$
f=\psi h,
$$

where $h$ is supported in $\Sigma_{1}$. Since $f \in \operatorname{Ham}(\Sigma)$ and $\psi \in \operatorname{Ham}\left(\Pi_{\frac{7}{8}}\right)$, we get that $h$ is Hamiltonian in $\Sigma$. Since $H_{\text {comp }}^{1}\left(\Sigma_{1}, \mathbf{R}\right)$ embeds in $H_{\text {comp }}^{1}(\Sigma, \mathbf{R})$, it means that $h$ actually belongs to $\operatorname{Ham}\left(\Sigma_{1}\right)$.

Define a neighborhood $\mathscr{V}(\epsilon)$ of the identity in $\operatorname{Ham}(\Sigma)$ by the following condition: $f \in \mathscr{V}(\epsilon)$, if, first, $\|\psi\|<\epsilon$ (recall that when $f$ converges to the identity, so does $\psi$ ) and, second, $h \in \mathscr{V}_{1}(\epsilon)$. Hence, if $f \in \mathscr{V}(\epsilon)$, we can write it as a product of $N_{1}(\epsilon)+1$ diffeomorphisms $g_{i}$, where each $g_{i}$ is $\epsilon$-close to the identity and belongs to $\operatorname{Ham}\left(D_{j}\right)$ for some disc $D_{j} \subset \Sigma$. This proves the claim $(*)$ for $\Sigma$ in the first case.

Second case. The surface $\Sigma$ is closed - we view it as a result of gluing a disc to a surface $\Sigma_{0}$ with one boundary component. Choose a diffeomorphism $\varphi: \mathbb{D}^{2} \rightarrow \overline{\Sigma-\Sigma_{0}}$ sending the boundary of $\mathbb{D}^{2}$ into the boundary of $\Sigma_{0}$. Denote by $D_{r}$ the image by $\varphi$ of the disc of radius $r \in[0,1]$ in $\mathbb{D}^{2}$. Let $\mathscr{V}_{1}(\epsilon)$ be the neighborhood of the identity given by $(*)$ applied to the surface $\Sigma_{1}:=\Sigma_{0} \cup \varphi\left(\left\{z \in \mathbb{D}^{2},|z| \geq \frac{1}{4}\right\}\right)$ and let $N_{1}(\epsilon)$ be the corresponding integer - recall that in the first case above we have already proved $(*)$ for $\Sigma_{1}$, which is a surface with boundary.

Let $f \in \operatorname{Ham}(\Sigma),\|f\|<\epsilon$. We apply Lemma 6.2 to the chain of discs $D_{\frac{1}{2}} \subset D_{\frac{3}{4}} \subset D_{1}$ and to the restriction of $f$ to $D_{\frac{3}{4}}$. We obtain a diffeomorphism $\psi$ supported in $D_{1}$ and close to the identity which coincides with $f$ on $D_{\frac{1}{2}}$. Hence, we can write

$$
f=\psi h,
$$

where $h$ is supported in $\Sigma_{1}$. Since $f \in \operatorname{Ham}(\Sigma)$ and $\psi \in \operatorname{Ham}\left(D_{1}\right)$, we get that $h$ is Hamiltonian in $\Sigma$. Since $\Sigma_{1}$ has one boundary component, 
$H_{\text {comp }}^{1}\left(\Sigma_{1}, \mathbf{R}\right)$ embeds in $H_{\text {comp }}^{1}(\Sigma, \mathbf{R})$, so $h$ actually belongs to $\operatorname{Ham}\left(\Sigma_{1}\right)$. One concludes the proof as in the first case.

This finishes the proof of Theorem 3.1 (modulo the proofs of the extension lemmas).

\subsection{Extension lemmas}

The area-preserving lemmas for discs and rectangles will follow from the following:

Lemma 6.5 (Area-preserving extension lemma for annuli). Let $\mathbb{A}=S^{1} \times$ $[-3,3]$ be a closed annulus and let $\mathbb{A}_{1}=S^{1} \times[-1,1], \mathbb{A}_{2}=S^{1} \times[-2,2]$ be smaller annuli inside $\mathbb{A}$. Let $\phi$ be an area-preserving embedding of a fixed open neighborhood of $\mathbb{A}_{1}$ into $\mathbb{A}_{2}$ (we assume that $\mathbb{A}$ is equipped with some area form $\omega$ ), so that for some $y \in[-1,1]$ (and hence for all of them) the curves $S^{1} \times y$ and $\phi\left(S^{1} \times y\right)$ are homotopic in $\mathbb{A}$ and

$$
\text { the area in } \mathbb{A} \text { bounded by } S^{1} \times y \text { and } \phi\left(S^{1} \times y\right) \text { is } 0 \text {. }
$$

Then, there exists $\psi \in \operatorname{Ham}(\mathbb{A})$ such that $\left.\psi\right|_{\mathbb{A}_{1}}=\phi$ and $\|\psi\| \rightarrow 0$ as $\|\phi\| \rightarrow 0$.

Moreover, if for some arc $I \subset S^{1}$ we have that $\phi=\mathbb{1}$ outside a quadrilateral $I \times[-1,1]$ and $\phi(I \times[-1,1]) \subset I \times[-2,2]$, then $\psi$ can be chosen to be the identity outside $I \times[-3,3]$.

Let us show how this lemma implies the area-preserving extension lemmas for discs and rectangles.

\section{Proof of Lemma 6.2.}

Up to replacing $D_{2}$ by a slightly smaller disc, we can assume that $\phi$ is defined in a neighborhood of $D_{2}$. Identify some small neighborhood of $\partial D_{2}$ with $\mathbb{A}=S^{1} \times[-3,3]$ so that $\partial D_{2}$ is identified with $S^{1} \times 0 \subset \mathbb{A}_{1} \subset \mathbb{A}_{2} \subset \mathbb{A}$ and $\phi\left(\mathbb{A}_{1}\right) \subset$ Interior $\left(\mathbb{A}_{2}\right) \subset \mathbb{A} \subset$ Interior $(D) \backslash \phi\left(D_{1}\right)$.

Apply Lemma 6.5 and find $h \in \operatorname{Ham}(\mathbb{A}),\|h\| \rightarrow 0$ as $\epsilon \rightarrow 0$, so that $\left.h\right|_{\mathbb{A}_{1}}=\phi$. Set $\phi_{1}:=h^{-1} \circ \phi \in \operatorname{Ham}(D)$. Note that $\left.\phi_{1}\right|_{D_{1}}=\phi$ and $\phi_{1}$ is the identity on $\mathbb{A}_{1}$. Therefore we can extend $\left.\phi_{1}\right|_{D_{2} \cup \mathbb{A}_{1}}$ to $D$ by the identity and get the required $\psi$.

\section{Proof of Lemma 6.3.}


Identify the rectangles $\Pi_{1} \subset \Pi_{2} \subset \Pi$ - by a diffeomorphism - with quadrilaterals $I \times[-1,1] \subset I \times[-2,2] \subset I \times[-3,3]$ in the annulus $\mathbb{A}=$ $S^{1} \times[-3,3]$ for some suitable arc $I \subset S^{1}$ and apply Lemma 6.5.

In order to prove Lemma 6.5, we first need to prove a version of the lemma concerning smooth (not necessarily area-preserving) embeddings.

Lemma 6.6 (Smooth extension lemma). Let $\mathbb{A}_{1} \subset \mathbb{A}_{2} \subset \mathbb{A}$ be as in Lemma 6.5. Let $\phi$ be a smooth embedding of a fixed open neighborhood of $\mathbb{A}_{1}$ into $\mathbb{A}_{2}$, isotopic to the identity, such that $\|\phi\| \leq \epsilon$ for some $\epsilon>0$. Then there exists $\psi \in \operatorname{Diff}_{0, c}(\mathbb{A})$ such that $\psi$ is supported in $\mathbb{A}_{2},\left.\psi\right|_{\mathbb{A}_{1}}=\phi$, and $\|\psi\| \leq C \epsilon$, for some $C>0$, independent of $\phi$.

Moreover, if $\phi=\mathbb{1}$ outside a quadrilateral $I \times[-1,1]$ and $\phi(I \times[-1,1]) \subset$ $I \times[-2,2]$ for some arc $I \subset S^{1}$, then $\psi$ can be chosen to be the identity outside $I \times[-3,3]$.

Lemma 6.6 will be proved in Section 6.4.

\section{Proof of Lemma 6.5.}

As one can easily check using Proposition 6.1, we can assume without loss of generality that the area form on $\mathbb{A}=S^{1} \times[-3,3]$ is $\omega=d x \wedge d y$, where $x$ is the angular coordinate along $S^{1}$ and $y$ is the coordinate along $[-3,3]$. All norms and distances are measured with the Euclidean metric on $\mathbb{A}$.

Denote $\mathbb{A}_{+}:=S^{1} \times[1,2], \mathbb{A}_{-}:=S^{1} \times[-2,-1]$.

Assume $\|\phi\|<\epsilon$. By Lemma 6.6, there exists $f \in \operatorname{Diff}_{0, c}\left(\mathbb{A}_{2}\right)$ such that $\|f\| \leq C \epsilon$, and $f=\phi$ on a neighborhood of $\mathbb{A}_{1}$. Denote $\Omega:=f^{*} \omega$. By $(2)$,

$$
\int_{\mathbb{A}_{+}} \Omega=\int_{\mathbb{A}_{+}} \omega, \int_{\mathbb{A}_{-}} \Omega=\int_{\mathbb{A}_{-}} \omega .
$$

Note that $\Omega$ coincides with $\omega$ on a neighborhood of $\partial \mathbb{A}_{+}$and $\partial \mathbb{A}_{-}$. Let us find $h \in \operatorname{Diff}_{0, c}\left(\mathbb{A}_{2}\right)$ such that

- $\left.h\right|_{\mathbb{A}_{1}}=11$,

- $h^{*} \Omega=\omega$,

- $\|h\| \rightarrow 0$ as $\epsilon \rightarrow 0$.

Given such an $h$, we extend $f h$ by the identity to the whole of $\mathbb{A}$. The resulting diffeomorphism of $\mathbb{A}$ is $C^{0}$-small (if $\epsilon$ is sufficiently small), preserves 
$\omega$ and belongs to $\operatorname{Diff}_{0, c}(\mathbb{A})$, hence (see e.g. [49]), also to $\mathcal{D}(\mathbb{A})$. It may not be Hamiltonian but one can easily make it Hamiltonian by a $C^{0}$-small adjustment on $\mathbb{A} \backslash \mathbb{A}_{2}$. The resulting diffeomorphism $\psi \in \operatorname{Ham}(\mathbb{A})$ will have all the required properties.

\section{Preparations for the construction of $h$.}

Since on $\mathbb{A}_{1}$ the map $h$ is required to be identity, we need to construct it on $\mathbb{A}_{+}$and $\mathbb{A}_{-}$. We will construct $h_{+}:=\left.h\right|_{\mathbb{A}_{+}}$, the case of $\mathbb{A}_{-}$is similar. By a rectangle or a square in $\mathbb{A}$ we mean the product of a connected arc in $S^{1}$ and an interval in $[-3,3]$.

Let us divide $\mathbb{A}_{+}=S^{1} \times[1,2]$ into closed squares $K_{1}, \ldots, K_{N}$, with a side of size $r=\epsilon^{1 / 4}>3 \epsilon$ (we assume that $\epsilon$ is sufficiently small). Denote by $V$ the set of the vertices which are not on the boundary and by $E$ the set of the edges which are not on the boundary. Finally, denote by $\Gamma$ the 1 -skeleton of the partition (i.e. the union of all the edges).

For each $v \in V$ denote by $B_{v}(\delta)$ the open ball in $\mathbb{A}_{+}$of radius $\delta>0$ with the center at $v$. Fix a small positive $\delta_{0}<r$ so that for $0<\delta<\delta_{0}$, the balls $B_{v}(\delta), v \in V$, are disjoint and each $B_{v}(\delta)$ intersects only the edges adjacent to $v$. Given such a $\delta$, consider for each edge $e \in E$ a small open rectangle $U_{e}(\delta)$ covering $e \backslash\left(e \cap \cup_{v \in V} B_{v}(\delta)\right)$, so that

- $U_{e}(\delta) \cap B_{v}(\delta) \neq \emptyset$ if and only if $v$ is adjacent to $e$.

- $U_{e}(\delta)$ does not intersect any other edge apart from $e$.

- All the rectangles $U_{e}(\delta), e \in E$, are mutually disjoint.

Define a neighborhood $U(\delta)$ of $\Gamma$ by

$$
U(\delta)=\left(\cup_{v \in V} B_{v}(\delta)\right) \cup\left(\cup_{e \in E} U_{e}(\delta)\right) .
$$

For each $\varepsilon_{1}>\varepsilon_{2}>0$ we pick a cut-off function $\chi_{\varepsilon_{1}, \varepsilon_{2}}: \mathbf{R} \rightarrow[0,1]$ which is equal to 1 on a neighborhood of $\left(-\varepsilon_{2}, \varepsilon_{2}\right)$ and vanishes outside $\left(-\varepsilon_{1}, \varepsilon_{1}\right)$. Finally, by $C_{1}, C_{2}, \ldots$ we will denote positive constants independent of $\epsilon$.

The construction of $h_{+}$will proceed in several steps.

\section{Adjusting $\Omega$ on $\Gamma$.}

We are going to adjust the form $\Omega$ by a diffeomorphism supported inside $U(\delta)$ to make it equal to $\omega$ on $\Gamma$. One can first construct $h_{1} \in \operatorname{Diff}_{0, c}\left(\mathbb{A}_{+}\right)$ supported in $\cup_{v \in V} B_{v}(2 \delta)$ such that $h_{1}^{*} \Omega=\omega$ on $\cup_{v \in V} B_{v}(\delta)$ for some $\delta<\delta_{0}$ 
(simply using Darboux charts for $\Omega$ and $\omega$ ). Note that $\left\|h_{1}\right\|<2 \delta$. Write $\Omega^{\prime}:=h_{1}^{*} \Omega$. For each $e \in E$ we will construct a diffeomorphism $h_{e}$ supported in $U_{e}(\delta)$ so that $h_{e}^{*} \Omega^{\prime}=\omega$ on $l:=U_{e}(\delta) \cap e$ (and thus on the whole $e$, since $\Omega^{\prime}$ already equals $\omega$ on each $\left.B_{v}(\delta)\right)$.

Without loss of generality, let us assume that $e$ does not lie on $\partial \mathbb{A}_{+}$(since $\Omega^{\prime}$ already coincides with $\omega$ there) and that $U_{e}(\delta)$ is of the form $(a, b) \times(-\delta, \delta)$. Write the restriction of $\Omega^{\prime}$ on $l=(a, b) \times 0$ as $\beta(x) d x \wedge d y, \beta(x)>0$.

Consider a cut-off function $\chi=\chi_{\delta, \delta / 2}: \mathbf{R} \rightarrow[0,1]$ and define a vector field $\mathbf{w}(x, y)$ on $U_{e}(\delta)$ by

$$
\mathbf{w}(x, y)=\chi(y) \log (\beta(x)) y \frac{\partial}{\partial y} .
$$

Note that $\mathbf{w}=0$ on $l$ and has compact support in $U_{e}(\delta)$ (the end-points of $l$ lie in the balls $B_{v}(\delta)$ on which $\Omega=\omega$ and thus $\beta=1$ near these endpoints). Let $\varphi_{t}$ be the flow of $\mathbf{w}$. A simple calculation shows that

$$
\frac{d}{d t} \varphi_{t}^{*} \omega=\varphi_{t}^{*} L_{\mathbf{w}} \omega=\log (\beta(x)) e^{t \log (\beta(x))} d x \wedge d y
$$

at the point $\varphi_{t}((x, 0))=(x, 0)$. Therefore $\varphi_{1}^{*} \omega=\Omega^{\prime}$ on $l$. Thus setting $h_{e}:=\varphi_{1}^{-1}$ we get that $h_{e}^{*} \Omega^{\prime}=\omega$ on $l$ and that $\left\|h_{e}\right\| \leq 2 \delta$, because $h_{e}$ preserves the fibers $x \times(-\delta, \delta)$. Set

$$
h_{2}:=\prod_{e \in E} h_{e} .
$$

Since the rectangles $U_{e}(\delta)$ are pairwise disjoint, $h_{2}$ is supported in $U(\delta)$ and satisfies the conditions

- $h_{2}^{*} \Omega^{\prime}=\omega$ on $\Gamma$.

- $\left\|h_{2}\right\| \leq 2 \delta$.

The diffeomorphism $h_{3}:=h_{2} h_{1} \in \operatorname{Diff}_{0, c}\left(\mathbb{A}_{+}\right)$satisfies $\left\|h_{3}\right\| \leq 4 \delta$ and

$$
h_{3}^{*} \Omega=h_{2}^{*} \Omega^{\prime}=\omega \text { on } \Gamma .
$$

Consider the area form $\Omega^{\prime \prime}:=h_{3}^{*} \Omega$. It coincides with $\omega$ on the 1 -skeleton $\Gamma$ and near $\partial \mathbb{A}_{+}$. Moreover, $\int_{\mathbb{A}_{+}} \Omega^{\prime \prime}=\int_{\mathbb{A}_{+}} \Omega^{\prime}$ and hence, by (3),

$$
\int_{\mathbb{A}_{+}} \Omega^{\prime \prime}=\int_{\mathbb{A}_{+}} \omega .
$$




\section{Adjusting the areas of the squares}

In this paragraph we construct a $C^{0}$-perturbation $\rho \omega$ of $\omega$ which has the same integral as $\Omega^{\prime \prime}$ on each square $K_{i}$.

Making $\delta$ sufficiently small we can assume that $\left\|h_{3}\right\|<\epsilon$. Recall that $r=\epsilon^{1 / 4}>3 \epsilon$. Therefore the image of one of the squares $K_{i}$ by $h_{3}$ contains a square of area $(r-\epsilon)^{2}$ and is contained in a square of area $(r+\epsilon)^{2}$. Hence,

$$
\frac{(r-2 \epsilon)^{2}}{r^{2}} \leq \frac{\int_{K_{i}} \Omega^{\prime \prime}}{\int_{K_{i}} \omega} \leq \frac{(r+2 \epsilon)^{2}}{r^{2}} .
$$

Since $\epsilon / r=\epsilon^{3 / 4} \rightarrow 0$ as $\epsilon \rightarrow 0$, we get that if $\epsilon$ is sufficiently small, there exists $C_{1}>0$ so that

$$
1-C_{1} \frac{\epsilon}{r} \leq \frac{\int_{K_{i}} \Omega^{\prime \prime}}{\int_{K_{i}} \omega} \leq 1+C_{1} \frac{\epsilon}{r} .
$$

Now set $s_{i}:=\int_{K_{i}} \Omega^{\prime \prime}$ and $t_{i}=s_{i} / r^{2}-1$. By (5),

$$
\left|t_{i}\right| \leq C_{1} \frac{\epsilon}{r}=C_{1} \epsilon^{3 / 4}
$$

For each $i$ we can choose a nonnegative function $\bar{\rho}_{i}$ supported in the interior of $K_{i}$ so that $\int_{K_{i}} \bar{\rho}_{i} \omega=r^{2}$ and

$$
\left\|\bar{\rho}_{i}\right\|_{C^{2}} \leq C_{2} \epsilon^{-1 / 2}
$$

for some constant $C_{2}>0$ independent of $i$. Define a function $\varrho$ on $\mathbb{A}$ by

$$
\varrho:=1+\sum_{i=1}^{N} t_{i} \bar{\rho}_{i} .
$$

By (6) and (7), the function $\varrho$ is positive and the form $\varrho \omega$ converges to $\omega$ (in the $C^{0}$-sense) as $\epsilon$ goes to 0 . Moreover, $\varrho$ is equal to 1 on $\Gamma$ and the two area forms $\varrho \omega$ and $\Omega^{\prime \prime}$ have the same integral on each $K_{i}$. By (4), one has:

$$
\int_{\mathbb{A}_{+}} \varrho \omega=\int_{\mathbb{A}_{+}} \Omega^{\prime \prime}=\int_{\mathbb{A}_{+}} \omega .
$$

\section{Finishing the construction of $h_{+}$: Moser's argument.}


Let us apply Proposition 6.1, part (ii), to the forms $\Omega^{\prime \prime}$ and $\varrho \omega$ on $\mathbb{A}_{+}$: these forms have the same integral over each $K_{i}$ and coincide on $\Gamma$ and near the boundary of $\mathbb{A}_{+}$, therefore there exists a diffeomorphism $h_{4} \in \operatorname{Diff}{ }_{0, c}\left(\mathbb{A}_{+}\right)$ which is the identity on $\Gamma$ and satisfies $h_{4}^{*} \Omega^{\prime \prime}=\varrho \omega$. Since $h_{4}$ is the identity on $\Gamma$ and maps each $K_{i}$ into itself, its $C^{0}$-norm is bounded by the diameter of $K_{i}$, hence goes to 0 with $\epsilon$.

Finally, apply Proposition 6.1 to the forms $\omega$ and $\varrho \omega$ on $\mathbb{A}_{+}$: by (8), their integrals over $\mathbb{A}_{+}$are the same, they coincide on $\partial \mathbb{A}_{+}$and are $C^{0}$-close. Therefore there exists $h_{5} \in \operatorname{Diff}_{0, c}\left(\mathbb{A}_{+}\right)$so that $h_{5}^{*}(\varrho \omega)=\omega$ and

$$
\left\|h_{5}\right\| \rightarrow 0 \text { as } \epsilon \rightarrow 0 .
$$

Then $h_{+}:=h_{5} h_{4} h_{3}$ is the required diffeomorphism. This finishes the construction of $h$.

\section{Final observation.}

Note that if $\phi=\mathbb{1}$ outside a quadrilateral $I \times[-1,1]$ for some $\operatorname{arc} I \subset S^{1}$, then $f$ can be chosen to have the same property. In such a case we need to construct $h_{+} \in \operatorname{Diff}_{0, c}\left(\mathbb{A}_{+}\right)$supported in $I \times[-3,3]$.

Let $J$ be the complement of the interval $I$ in the circle. The partition of $\mathbb{A}_{+}$into squares can be chosen so that it extends a partition of $J \times[1,2] \subset \mathbb{A}_{+}$ into squares of the same size. Going over each step of the construction of $h_{+}$ above, we see that, since $\Omega=\omega$ on $J \times[1,2]$, each of the maps $h_{1}, h_{2}, h_{3}, h_{4}, h_{5}$ can be chosen to be identity on each of the squares in $J \times[1,2]$, hence on the whole $J \times[1,2]$. Therefore $h_{+}$, hence $h$, hence $\psi=f h$, is the identity on $J \times[1,2]$. Moreover $\psi$ is automatically Hamiltonian in this case.

\subsection{Proof of the smooth extension lemma}

As in the proof of Lemma 6.5, we assume that the Riemannian metric on $\mathbb{A}=S^{1} \times[-3,3]$ used for the measurements is the Euclidean product metric. We can also assume that the neighborhood of $\mathbb{A}_{1}$ on which $\phi$ is defined is, in fact, an open neighborhood of $\mathbb{A}^{\prime}:=S^{1} \times[-1.5,1.5]$ and that $\epsilon \ll 0.5$.

\section{Proof of Lemma 6.6.}

Applying Lemma 7.1 (see the appendix by M. Khanevsky below) to the two curves $S^{1} \times\{ \pm 1.5\}$ and their images under $\phi$ we can find $\psi_{1} \in \operatorname{Diff}_{0, c}(\mathbb{A})$, supported in $S^{1} \times(-2,-1) \cup S^{1} \times(1,2)$, such that $\psi_{1}$ coincides with $\phi^{-1}$ on the 
curves $\phi\left(S^{1} \times\{ \pm 1.5\}\right)$. Moreover it satisfies $\left\|\psi_{1}\right\|<C^{\prime} \epsilon$. Define $\psi_{2}:=\psi_{1} \phi$. This map is defined on an open neighborhood of $\mathbb{A}^{\prime}=S^{1} \times[-1.5,1.5]$ and has the following properties:

- The restriction of $\psi_{2}$ to $\mathbb{A}^{\prime}$ is a diffeomorphism of $\mathbb{A}^{\prime}$. It is the identity on $\partial \mathbb{A}^{\prime}$ and coincides with $\phi$ on $\mathbb{A}_{1}=S^{1} \times[-1,1] \subset \mathbb{A}^{\prime}$.

- $\left\|\psi_{2}\right\|<C^{\prime \prime} \epsilon$, where $C^{\prime \prime}:=C^{\prime}+1$.

We are going to modify $\psi_{2}$ (by a $C^{0}$-small perturbation) to make it the identity not only on $\partial \mathbb{A}^{\prime}$ but on an open neighborhood of $\partial \mathbb{A}^{\prime}$. Then we will extend it by the identity to a diffeomorphism of $\mathbb{A}$ with the required properties.

Since $\psi_{2}$ is the identity on $\partial \mathbb{A}^{\prime}$, by perturbing it slightly near $\partial \mathbb{A}^{\prime}$ (in the $C^{\infty}$-norm) we can assume that, in addition to the properties listed above, near $\partial \mathbb{A}^{\prime}$ the map $\psi_{2}$ preserves the foliation of $\mathbb{A}$ by the circles $S^{1} \times y$. It means that for some sufficiently small $r>0$ the restriction of $\psi_{2}$ to $S^{1} \times$ $[-1.5,-1.5+r] \cup S^{1} \times[1.5-r, 1.5]$ has the form

$$
\psi_{2}:(x, y) \mapsto(x+u(x, y), y),
$$

for some smooth function $u$ such that $\|u\|<C^{\prime \prime} \epsilon$. Choose a cut-off function $\chi=\chi_{1.5,1.5-r}: \mathbf{R} \rightarrow[0,1]$ and define a map $\psi_{3}$ on $\mathbb{A}^{\prime}$ as follows:

$$
\begin{gathered}
\psi_{3}:=\psi_{2} \text { on } S^{1} \times[-1.5+r, 1.5-r], \\
\psi_{3}(x, y):=(x+\chi(y) u(x, y), y), \text { when }|y| \geq 1.5-r .
\end{gathered}
$$

We now consider the diffeomorphism $\psi$ which equals $\psi_{3}$ on $\mathbb{A}^{\prime}$ and the identity outside $\mathbb{A}^{\prime}$. It coincides with $\phi$ on $\mathbb{A}_{1}$ and satisfies $\|\psi\|<C^{\prime \prime} \epsilon$. Note that if $\epsilon$ is sufficiently small, $\psi$ automatically belongs to the identity component $\operatorname{Diff}_{0, c}(\mathbb{A})$ (this can be easily deduced, for instance, from [15, 16] or [48]). This finishes the construction of $\psi$ in the general case.

Let us now consider the case, where $\phi=\mathbb{1}$ outside a quadrilateral $I \times$ $[-1,1]$ and $\phi(I \times[-1,1]) \subset I \times[-2,2]$ for some arc $I \subset S^{1}$. Then, by Lemma 7.1, we can assume that $\psi_{1}$ is supported in $I \times[-3,3]$. Then $\psi_{2}$ is the identity outside $I \times[-1.5,1.5]$. When we perturb $\psi_{2}$ near $\partial \mathbb{A}^{\prime}$ to make it preserve the foliation by circles, we can choose the perturbation to be supported in $I \times[-1.5,1.5]$. Thus $u(x, y)$ would be 0 outside $I \times[-1.5,1.5]$. This yields that $\psi_{3}$, and consequently $\psi$, are the identity outside $I \times[-3,3]$. 


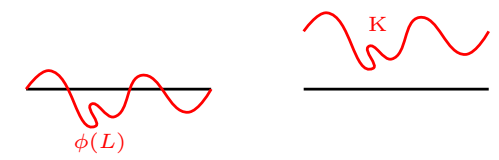

Figure 1: Shifting $L$

\section{Appendix by Michael Khanevsky: An extension lemma for curves}

For a diffeomorphism $\phi$ of a compact surface with a Riemannian distance $d$ we write $\|\phi\|=\max d(x, \phi(x))$. The purpose of this appendix is to prove the following extension lemma which was used in Section 6.4 above.

Lemma 7.1. Let $A:=S^{1} \times[-1,1]$ be an annulus equipped with the Euclidean product metric. Set $L=S^{1} \times 0$. Assume that $\phi$ is a smooth embedding of an open neighborhood of $L$ in $A$, so that $L$ is homotopic to $\phi(L)$ and $\|\phi\| \leq \epsilon$ for some $\epsilon \ll 1$.

Then there exists a diffeomorphism $\psi \in \operatorname{Diff}_{0, c}(A)$ such that $\psi=\phi$ on $L$ and $\|\psi\|<C^{\prime} \epsilon$ for some $C^{\prime}>0$ independent of $\psi$.

Moreover, if $\phi=11$ outside some arc $I \subset L$ and $\phi(I) \subset I \times[-1,1]$, then $\psi$ can be made the identity outside $I \times[-1,1]$.

Proof. We view the coordinate $x$ on $A$ along $S^{1}$ as a horizontal one, and the coordinate $y$ along $[-1,1]$ as a vertical one. If $a, b \in L$ are not antipodal, we denote by $[a, b]$ the shortest closed arc in $L$ between $a$ and $b$.

The proof consist of a few steps. By $C_{1}, C_{2}, \ldots$ we will denote some universal positive constants.

Step 1. Shift the curve $\phi(L)$ by $3 \epsilon$ upward by a diffeomorphism $\psi_{1} \in$ $\operatorname{Diff}_{0, c}(A)$ with $\left\|\psi_{1}\right\| \leq C_{1} \epsilon$, so that $K:=\psi_{1}(\phi(L))$ lies strictly above $L$ (see figure 1).

Step 2. Let $x_{1}, \ldots, x_{N}$ be points on $L$ chosen in a cyclic order so that the distance between any two consecutive points $x_{i}$ and $x_{i+1}$ is at most $\epsilon$ (here and further on, $i+1$ is taken to be 1 , if $i=N$ ). 

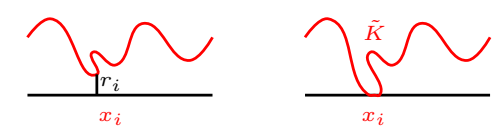

Figure 2: $\tilde{K}$ coincides with $L$ near $x_{i}$

For each $i=1, \ldots, N$, consider a vertical ray originating at $x_{i}$ and assume, without loss of generality, that it is transversal to $K$ and that $K$ is parallel to $L$ near its intersection points with the ray. Among the intersection points of the ray with $K$ choose the closest one to $L$ and denote it by $y_{i}$. Denote by $r_{i}$ the closed vertical interval between $x_{i}$ and $y_{i}$. Choose small pairwise disjoint open rectangles $U_{i}$, of width at most $\epsilon / 3$ and of height at most $4 \epsilon$, around each of the intervals $r_{i}$.

For each $i=1, \ldots, N$, it is easy to construct a diffeomorphism $\psi_{2, i}$ supported in $U_{i}$ which moves a connected arc of $K \cap U_{i}$ containing $y_{i}$ by a parallel shift downwards into an arc of $L$ containing $x_{i}$ so that $\psi_{2, i}(K)$ lies completely in $\{y \geq 0\}$. Set $\psi_{2}:=\prod_{i=1}^{N} \psi_{2, i}$. Clearly, $\left\|\psi_{2, i}\right\| \leq C_{2} \epsilon$ for each $i$ and therefore, since the supports of all the diffeomorphisms $\psi_{2, i}$ are disjoint, $\left\|\psi_{2}\right\| \leq C_{2} \epsilon$ as well. Set (see figure 2)

$$
\tilde{\psi}:=\psi_{2} \psi_{1} \in \operatorname{Diff}_{0, c}(A), \quad \tilde{K}:=\tilde{\psi}(\phi(L)) .
$$

Note that $\|\tilde{\psi}\| \leq C_{3} \epsilon$

Step 3. Note that the points $x_{i}, i=1, \ldots, N$, lie on $\tilde{K}$ and that

$$
\tilde{K} \subset\{y \geq 0\}
$$

An easy topological argument shows that in such a case, since the points $x_{i}$ lie on $L$ in cyclic order, they also lie in the same cyclic order on $\tilde{K}$.

For each $i$ there are two arcs in $\tilde{K}$ connecting $x_{i}$ and $x_{i+1}$ - denote by $K_{i}$ the one homotopic with fixed endpoints to the arc $\left[x_{i}, x_{i+1}\right] \subset L$. Since the points $x_{i}$ lie on $\tilde{K}$ in the same cyclic order as on $L$, we see that $K_{1}, \ldots, K_{N}$ are precisely the closures of the $N$ open arcs in $\tilde{K}$ obtained by removing the points $x_{1}, \ldots, x_{N}$ from $\tilde{K}$.

Let $B_{i}$ be the open set bounded by $K_{i}$ and $\left[x_{i}, x_{i+1}\right]$ (see figure 3 ). The $B_{i}$ 's are disjoint and have diameter at most $C_{4} \epsilon$. Let $B_{i}^{\prime}$ be disjoint open neighborhoods of the $B_{i}$ 's of diameter at most $C_{5} \epsilon$. Now for each $i$ one can 


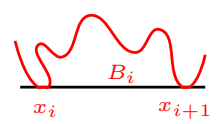

Figure 3: The open set $B_{i}$

easily find a diffeomorphism $\psi_{3, i} \in \operatorname{Diff}_{0, c}\left(B_{i}^{\prime}\right)$ such that $\left\|\psi_{3, i}\right\| \leq C_{5} \epsilon$ and $\psi_{3, i}\left(K_{i}\right)=\left[x_{i}, x_{i+1}\right]$. Set $\psi_{3}:=\prod_{i=1}^{N} \psi_{3, i}$. Since the supports of all $\psi_{3, i}$ are pairwise disjoint we get that $\left\|\psi_{3}\right\| \leq C_{5} \epsilon$.

Step 4. Define $\psi_{4}:=\psi_{3} \tilde{\psi}=\psi_{3} \psi_{2} \psi_{1}$. Clearly, $\psi_{4} \in \operatorname{Diff}_{0, c}(A)$ and $\left\|\psi_{4}\right\| \leq$ $C_{6} \epsilon$. Recall that for each $i$ we have $\psi_{3}\left(K_{i}\right)=\left[x_{i}, x_{i+1}\right]$ and that each $K_{i}$ is the shortest arc between $x_{i}$ and $x_{i+1}$ in $\tilde{K}=\psi_{2} \psi_{1}(L)$. Thus $\psi_{4}$ maps $K$ into $L$. The diffeomorphism $\psi_{4}^{-1}$ satisfy $\psi_{4}^{-1}(L)=\phi(L)$. We now obtain easily the required $\psi$ by a $C^{0}$-small perturbation of $\psi_{4}^{-1}$.

\section{References}

[1] M. Akveld, D. Salamon, Loops of Lagrangian submanifolds and pseudoholomorphic discs, Geom. and Funct. Analysis 11, No. 4, (2001), 609650 .

[2] A. Banyaga, Sur la structure du groupe des difféomorphismes qui préservent une forme symplectique, Comm. Math. Helv. 53 (1978), 174227.

[3] A. Banyaga, Formes-volume sur les variétés à bord, Enseignement Math. (2) 20 (1974), 127-131.

[4] A. Banyaga, The structure of classical diffeomorphism groups, Mathematics and its applications 400, Kluwer Academic Publishers Group, Dordrecht, 1997.

[5] J. Barge, E. Ghys, Cocycles d'Euler et de Maslov, Math. Ann. 294 (1992), 235-265.

[6] C. Bavard, Longueur stable des commutateurs, Enseign. Math. (2) 37, No. 1-2 (1991), 109-150. 
[7] G. Ben Simon, The Nonlinear Maslov index and the Calabi homomorphism, Commun. Contemp. Math. 9, No. 6 (2007), 769-780.

[8] P. Biran, Connectedness of spaces of symplectic embeddings, Internat. Math. Res. Notices 10 (1996), 487-491.

[9] P. Biran, M. Entov, L. Polterovich, Calabi quasimorphisms for the symplectic ball, Commun. Contemp. Math. 6, No. 5 (2004), 793-802.

[10] A. Bounemoura, Simplicité des groupes de transformations de surfaces, Ensaios Matemáticos 14 (2008), 1-143.

[11] R. Brooks, Some remarks on bounded cohomology, in Riemann Surfaces and Related Topics: Proceedings of the 1978 Stony Brook Conference (State Univ. New York, Stony Brook, N.Y., 1978), 53-63, Ann. of Math. Stud. 97, Princeton University Press, Princeton, 1981.

[12] D. Burago, S. Ivanov, L. Polterovich, Conjugation-invariant norms on groups of geometric origin, in Groups of Diffeomorphisms: In Honor of Shigeyuki Morita on the Occasion of His 60th Birthday, Advanced Studies in Pure Mathematics 52, Math. Society of Japan, Tokyo, 2008.

[13] E. Calabi, On the group of automorphisms of a symplectic manifold, in Problems in analysis, 1-26, Princeton Univ. Press, Princeton, 1970.

[14] D. Calegari, scl, monograph in preparation, to appear in Memoirs MSJ, available from http:/www.its. caltech.edu/ dannyc.

[15] C.J. Earle, J. Eells, The diffeomorphism group of a compact Riemann surface, Bull. Amer. Math. Soc. 73 (1967), 557-559.

[16] C.J. Earle, J. Eells, A fibre bundle description of Teichmüller theory, J. Differential Geometry 3 (1969), 19-43.

[17] M. Entov, Commutator length of symplectomorphisms, Comment. Math. Helv. 79, No. 1 (2004), 58-104.

[18] M. Entov, L. Polterovich, Calabi quasimorphism and quantum homology, Int. Math. Res. Not. 30 (2003), 1635-1676. 
[19] M. Entov, L. Polterovich, Symplectic quasi-states and semi-simplicity of quantum homology, in Toric Topology, 47-70, Contemporary Mathematics 460, AMS, Providence, 2008.

[20] A. Fathi, Structure of the group of homeomorphisms preserving a good measure on a compact manifold, Ann. Sci. École Norm. Sup. (4) 13, No. 1 (1980), 45-93.

[21] J.-M. Gambaudo et É. Ghys, Enlacements asymptotiques, Topology 36, No. 6 (1997), 1355-1379.

[22] J.-M. Gambaudo and E. Ghys, Commutators and diffeomorphisms of surfaces, Ergodic Theory Dynam. Systems 24, No. 5 (2004), 1591-1617.

[23] E. Ghys, Knots and dynamics, in International Congress of Mathematicians, Vol. I, 247-277, Eur. Math. Soc., Zürich, 2007.

[24] M. Gromov, Pseudoholomorphic curves in symplectic manifolds, Invent. Math. 82 (1985), 307-347.

[25] H. Hofer, On the topological properties of symplectic maps, Proc. of the Royal Soc. of Edinburgh 115A, No. 1-2 (1990), 25-28.

[26] H. Hofer, Estimates for the energy of a symplectic map, Comment. Math. Helv. 68, No. 1 (1993), 48-72.

[27] D. Kotschick, What is... a quasi-morphism?, Notices Amer. Math. Soc. 51, No. 2 (2004), 208-209.

[28] D. Kotschick, Stable length in stable groups, in Groups of Diffeomorphisms: In Honor of Shigeyuki Morita on the Occasion of His 60th Birthday, Advanced Studies in Pure Mathematics 52, Math. Society of Japan, Tokyo, 2008.

[29] F. Lalonde, Isotopy of symplectic balls, Gromov's radius and the structure of ruled symplectic 4-manifolds, Math. Ann. 300 (1994), 273-296.

[30] F. Lalonde, D. McDuff, The geometry of symplectic energy, Ann. of Math. 141, No. 2 (1995), 349-371.

[31] F. Lalonde and D. McDuff, Hofer's $L^{\infty}$-geometry: energy and stability of Hamiltonian flows I, Invent. Math. 122, No. 1 (1995), 1-33. 
[32] F. Lalonde and L. Polterovich, Symplectic diffeomorphisms as isometries of Hofer's norm, Topology 36, No. 3 (1997), 711-727.

[33] F. Le Roux, Six questions, a proposition and two pictures on Hofer distance for Hamiltonian diffeomorphisms on surfaces, preprint, 2007, available at http://www . umpa.ens-lyon.fr/ symplexe/publications.php.

[34] F. Le Roux, Simplicity of the group of compactly supported area preserving homeomorphisms of the open dics and fragmentation of symplectic diffeomorphisms, preprint, 2009, arXiv:0901.2428.

[35] D. McDuff, Remarks on the uniqueness of symplectic blowing up, in Symplectic geometry, D.Salamon ed., 157-167, London Math. Soc. Lecture Note Ser., 192, Cambridge Univ. Press, Cambridge, 1993.

[36] D. McDuff, From symplectic deformation to isotopy, in Topics in symplectic 4-manifolds (Irvine, CA, 1996), 85-99, First Int. Press Lect. Ser., I, Int. Press, Cambridge, MA, 1998.

[37] D. McDuff, D. Salamon, Introduction to symplectic topology, 2nd edition, Oxford University Press, Oxford, 1998.

[38] J. Moser, On the volume elements on a manifold, Trans. Amer. Math. Soc. 120 (1965), 288-294.

[39] Y.-G. Oh, $C^{0}$-coerciveness of Moser's problem and smoothing area preserving homeomorphisms, preprint, 2006, arXiv:math 0601183.

[40] Y. Ostrover, Calabi quasi-morphisms for some non-monotone symplectic manifolds, Algebr. Geom. Topol. 6 (2006), 405-434.

[41] L.Polterovich, Symplectic displacement energy for Lagrangian submanifolds, Ergodic Th. and Dynam. Syst. 13, No. 2 (1993), 357-367.

[42] L. Polterovich, The geometry of the group of symplectic diffeomorphisms, Lectures in Mathematics, ETH Zürich, Birkhäuser, Basel, 2001.

[43] L. Polterovich, Floer homology, dynamics and groups, in Morse theoretic methods in nonlinear analysis and in symplectic topology, 417-438, NATO Sci. Ser. II Math. Phys. Chem. 217, Springer, Dordrecht, 2006. 
[44] P. Py, Quasi-morphismes et invariant de Calabi, Ann. Sci. École Norm. Sup. (4) 39, No. 1 (2006), 177-195.

[45] P. Py, Quasi-morphismes de Calabi et graphe de Reeb sur le tore, C. R. Math. Acad. Sci. Paris 343, No. 5 (2006), 323-328.

[46] A. Shtern, Remarks on pseudocharacters and the real continuous bounded cohomology of connected locally compact groups, Ann. Global Anal. Geom. 20, No. 3 (2001), 199-221.

[47] J.-C. Sikorav, Approximation of a volume-preserving homeomorphism by a volume-preserving diffeomorphism, preprint, 2007, available at http://www . umpa.ens-lyon.fr/ symplexe/publications.php.

[48] S. Smale, Diffeomorphisms of the 2-sphere, Proc. Amer. Math. Soc. 10 (1959), 621-626.

[49] T. Tsuboi, The Calabi invariant and the Euler class, Trans. Amer. Math. Soc. 352, No. 2, (2000), 515-524.

Michael Entov

Department of Mathematics

Technion - Israel Institute of Technology

Haifa 32000, Israel

entov@math.technion.ac.il

Pierre Py

Department of Mathematics

University of Chicago

Chicago, Il 60637, USA

pierre.py@math.uchicago.edu
Leonid Polterovich

School of Mathematical Sciences

Tel Aviv University

Tel Aviv 69978, Israel

polterov@post.tau.ac.il

Michael Khanevsky

School of Mathematical Sciences

Tel Aviv University

Tel Aviv 69978, Israel

khanev@post.tau.ac.il 